\title{
Imaging In Vivo
}




\section{Abstract:}

24 Understanding mechanosensation and other sensory behavior in genetic model systems such as

25 C. elegans is relevant to many human diseases. These studies conventionally require

26 immobilization by glue and manual delivery of stimuli, leading to low experimental throughput

27 and high variability. Here we present a microfluidic platform that delivers precise mechanical

28 stimuli robustly. The system can be easily used in conjunction with functional imaging and

29 optical interrogation techniques, as well as other capabilities such as sorting or more

30 sophisticated fluid delivery schemes. The platform is fully automated, thereby greatly enhancing

31 the throughput and robustness of experiments. We show that behavior of the well-known gentle

32 and harsh touch neurons and their receptive fields can be recapitulated in our system. Using

33 calcium dynamics as a readout, we demonstrate the ability to perform a drug screen in vivo.

34 Furthermore, using an integrated chip platform that can deliver both mechanical and chemical

35 stimuli, we examine sensory integration in interneurons in response to multimodal sensory

36 inputs. We envision that this system will be able to greatly accelerate the discovery of genes and

37 molecules involved in mechanosensation and multimodal sensory behavior, as well as the

38 discovery of therapeutics for related diseases. 


\section{Introduction}

Mechanosensation is required for multiple sensory modalities such as touch, hearing, and balance, and is linked to a multitude of disorders including deafness ${ }^{1-4}$. Molecular mechanisms for mechanotransduction have been partially elucidated using a variety of model organisms, including Caenorhabditis elegans ${ }^{5-16}$. Conventional mechanosensation experiments with $C$. elegans typically involve the manual delivery of a mechanical stimulus to anterior or posterior regions of animals via an eyebrow hair or metal pick ${ }^{5,17-19}$, and visual scoring of touch avoidance behavior, an assay subject to considerable variability between experimenters. Computercontrolled stimulation methods, for example using a piezo-driven micro stylus, have been used with electrophysiological and functional imaging approaches to deliver more repeatable mechanical stimuli to animals ${ }^{20,21}$. However, recording of neuronal responses by patch clamping or calcium imaging in response to precisely controlled mechanical stimulation requires animals to be immobilized with glue ${ }^{15,20,21}$, limiting experimental throughput and disallowing the recovery of animals for screens or further experimentation. Moreover, gluing itself is likely to affect neuronal or circuit response, and differences in the extent of gluing introduce additional experimental variability.

Microfluidics has long been used as a "lab-on-a-chip" technology, allowing for wellcontrolled and high-throughput experiments with small samples ${ }^{22}$. In addition to enabling precise perturbations on the micron scale, microfluidic devices can easily be designed to work together with optical microscopy, allowing for imaging of fluorescent probes such as calcium indicators. For C. elegans experimentation particularly, microfluidics has been a widely adopted technology due to the match in length scale and compatibility with fluid handling ${ }^{23}$. Various devices have

63 been developed for delivering a variety of stimuli, including chemical cues and temperature 64 gradients, while simultaneously recording neuronal responses through calcium imaging ${ }^{24-33}$. In contrast, there are currently no microfluidic devices capable of delivering mechanical stimuli to C. elegans, or do so while recording neuronal activities in a controlled manner. In this work we present a microfluidic platform for delivering robust and precise mechanical stimuli to $C$. elegans by using pneumatically actuated structures. The device is fully automated, minimizing human variability and improving experimental throughput; it is fully compatible with fluorescent imaging of calcium dynamics of neurons, which enables mechanistic interrogations as well as 
71 high-throughput genetic or drug screens. Furthermore, the mechanical stimulus module of the

72 device can be easily integrated with other microfluidic modalities, allowing for multimodal

73 stimulation for sensory integration studies. Here we demonstrate the design and utility of such a

74 system in the context of high-throughput screening, as well as interrogate circuit dynamics in

75 multimodal sensory behavior.

\section{Results}

Our microfluidic device is optimized to deliver precise and repeatable mechanical stimuli to different anatomical regions of C. elegans (Fig. 1). After animals are loaded into an imaging channel (where the animals are not immobilized but their movement is much reduced from freely moving behavior), mechanical stimuli are delivered through two pairs of in-plane PDMS membrane structures (Fig. 1a and Supplementary Fig. 1). The structures are pressure-actuated, and when deflected, exert a mechanical stimulus on animals trapped in the imaging channel. The deflection and deformation caused by these actuations are in similar ranges as conventional approaches (Supplementary Fig. 2) 20,21 $^{2}$ Two additional actuated structures act as loading and imaging valves (Fig. 1a). This design retains animals in plane and relatively stationary but not fully immobilized, thus allowing high-quality imaging of calcium transients in cell bodies and subcellular processes (Fig. 1b). To automatically identify the fluorescently labeled neuron of interest and extract quantitative calcium transients, we developed a neuron tracking algorithm (Supplementary Fig. 3). The actuated structures are connected to a pressure source via individually controlled off-chip solenoid valves, allowing for an automated and rapid "load-andimage" routine (Fig. 1c). Additionally, the duration and pressure of stimuli can be easily controlled, allowing for the study of a variety of behaviors upon mechanical stimuli such as graded response, habituation, and arousal. Furthermore, this design can be easily adapted to allow for sorting and imaging animals of various sizes.

To demonstrate the utility of the system, we examined the responses of the classic gentle (AVM, ALMR/L, PVM, and PLMR/L) and harsh (PVD) touch receptor neurons ${ }^{19}$ (Fig. 1d). The stimulus is traditionally delivered to moving worms by a metal pick ${ }^{19}$, or to immobilized worms by a stiff probe ${ }^{15,20}$. By imaging calcium transients in animals expressing the genetically encoded calcium indicator (GECI) GCaMP6m ${ }^{34}$ in these touch receptor neurons, we show that the same 
102

103

104

105

106

107

108

109

110

111

112

113

114

115

116

117

118

119

120

121

122

123

124

125

126

127

128

129

130

131

132

device can deliver stimuli capable of exciting both the gentle and harsh touch neurons. Upon delivery of either a 1- or 2-second stimulus, calcium levels in cell bodies as well as in neuronal processes of both the gentle and harsh touch neurons rose as expected (Fig. 1e-m and Supplementary Movie 1-4). Spatially, these responses were consistent with the individual neurons' receptive fields as defined by anatomical and/or calcium imaging data ${ }^{5,18,20,35}$. AVM responded to anterior but not posterior stimuli (Fig. 1k). In contrast, PVD responded to both anterior and posterior stimuli, as did PVM, with the responses to posterior stimuli being stronger for both of these classes of neurons (Fig. 11, m). These results demonstrate that the mechanosensory chip delivers biologically relevant, spatially well-defined stimuli.

Because our system delivers mechanical stimuli by applying externally controlled pressure to actuated structures, the stimuli can be regulated by the magnitude and duration of the applied pressure (Fig. 2 and Supplementary Fig. 2). In the range of stimuli of relevance, the deformation in the animal tissue is roughly linear to the actuation pressure (Supplementary Fig. 2). To examine the effects of these two parameters, we applied anterior stimuli of varying levels of pressure and durations, and measured calcium activity in AVM neurons (Fig. 2a-d and Supplementary Fig. 4a, 5). Peak calcium transients were roughly proportional to the pressure applied (Fig. 2a, c) and the stimulus duration (Fig. 2b, d and Supplementary Fig. 4, 5). We also tested AVM's responses in the well-known mec-4/DEG/ENaC channel mutant (Fig. 2e). As expected, the mec-4 mutant gives negligible response and is insensitive to the magnitude of the stimulation input in the gentle touch regime, but is responsive to harsh touch, perhaps even more so than wild-type (Fig. 2e,f). Similarly, in the harsh touch regime, we presented posterior stimuli of varying pressure and durations, and observed responses in PVD neurons (Fig. 2g, h). As expected, compared to gentle touch neurons, PVD required higher pressure (55 psi) or longer duration of stimulus (5s) at low pressure to elicit similar responses. Furthermore, PVD also shows graded response to pressure and duration (Fig. 2g, h and Supplementary Fig. 4b).

Interestingly, in addition to response magnitude, the response rates of both the gentle touch and the harsh touch neurons are also functions of the stimulation pressure and duration (Fig. 2i). For AVM, stimuli with actuation pressure higher than 40 psi produce a response rate (fraction of animals responding) of $>90 \%$, while below 30 psi the response is more stochastic $(<20 \%)$ (Fig. 
$2 \mathrm{i}$ and Supplementary Fig. 4-6). Applying stimuli at lower actuation pressure also elicits a less sustained response or small magnitude of response and shorter stimuli elicits less response.

Besides simple stimulation, our system can also be used to deliver repeated stimuli in order to examine phenomena such as habituation and desensitization. Previous work has shown that presenting repeated mechanical stimuli can cause habituation in mechanosensory neurons ${ }^{20}$. To ask whether this phenomenon can be recapitulated in our system, we delivered repeated stimuli to animals using either short (1s) or long (3 min) inter-stimulus intervals (Fig. 2j-m and Supplementary Fig. 7). When receiving repeated stimuli with short intervals, the neurons exhibited an incremental increase in response magnitude up to the second stimuli, and then a reduced response in later stimuli (Fig. 2j, 1). In contrast, when using long inter-stimulus intervals, the response magnitude was reduced after each stimulus (Fig. 2k, m). These results are consistent with previous observations that habituation is dependent on inter-stimulus durations ${ }^{20}$. Thus, these experiments demonstrate how simple changes of operational parameters allow us to use the same device for a wider repertoire of the device utility.

In contrast to gluing protocols, our system allows for automated imaging by streamlining the handling of the worms; this in turn allows for high-throughput experiments that were not practicable before. To demonstrate the ability to perform rapid screens, we examined the effect of small molecules from an orphan ligand library on mechanosensation. We exposed animals to the compounds in L4 stage, and imaged AVM activity when delivering an anterior stimulus to adult worms (Fig. 3a). Figure 3b shows a typical response of wildtype animals without drug perturbation: calcium traces typically reach a maximum value shortly after the end of stimulus, and then slowly decline back to baseline levels. In order to examine how each drug affects mechanosensation, we quantitatively compared three metrics ( $\max \Delta \mathrm{R} / \mathrm{R}_{0}$, delay time, and halflife), as well as fraction of animals responding, between drug-treated animals and untreated animals (Fig. 3c-h and Supplementary Fig. 8, 9). We imaged adult animals exposed to 13 different drugs and quantified the established parameters for the screen criteria (Supplementary Table 1). While most of the drugs screened lowered the number of animals responding to mechanical stimulus, interestingly, a few drugs slightly increased the response fraction (Fig. 3c). We also analyzed differences in the metrics measure calcium dynamics for the drug treatment 
164

165

166

167

168

169

170

171

172

173

174

175

176

177

178

179

180

181

182

183

184

185

186

187

188

189

190

191

192

193

194

conditions, and found that five of the drugs we tested significantly affected mechanosensation response dynamics (Fig. 3d-f). Specifically, D-Alanine (\#5) and D-Arginine (\#8) significantly attenuated the $\max \Delta R / R_{0}$ while increasing the delay in the responses (Fig. 3d, e, h). In contrast, D-Lysine (\#4) only attenuated the max response (Fig. 3d, g). Two other drugs had effects only in the decay-time of response (Fig. 3f-h). D-Isoleucine (\#3) induced considerably smaller half-life for the calcium transients to return to baseline, much faster than in untreated animals (Fig. $3 f, g$ ). Lastly, $\beta$-Alanine (\#6) significantly increased the half-life, and calcium transients decreased in a slow linear gradient, instead of a typical exponential decay (Fig. 3f, h).

Another advantage of using microfluidics to deliver mechanosensory stimuli is that it is compatible with other microfluidic components to provoke additional sensory responses, e.g. chemosensation $^{25}$. C. elegans is a convenient system for studying multimodal sensory integration in vivo; worms have distinct sensory modalities such as mechanosensation and chemosensation, which allow them to find food sources and estimate danger. The difficulty to study sensory integration thus far is that there has not been a convenient method to integrate mechanosensory input with the existing tools, including microfluidic and optical methods ${ }^{23,25,36-38}$. With our mechanical stimulus device, incorporating chemosensory modules is readily attainable by simply adding channels to deliver chemical stimuli (Supplementary Fig. 10); without mechanical stimulation, the response of a chemosensory neuron to a chemical cue is as expected (Supplementary Fig. 11). To demonstrate the utility of the system for multimodal sensory integration, we focused on the response of the PVC command interneurons to both mechanical and chemical stimuli. PVC interneurons are postsynaptic to both the posterior mechanoreceptor neurons PLML/R, as well as the posterior chemosensory neurons PHBL/R, that have been shown to respond to $0.1 \%$ SDS stimulus ${ }^{5,39-41}$ (Fig. 4a and Supplementary Fig. 12a). Using our device to deliver multi-modal stimuli, we tested the ability of PVC to respond to stimuli within the same mode and cross-modality. When a single sub-threshold stimulus in either modality is delivered (i.e. 30s SDS stimulation to the tail, or 1s mechanical stimulation to the tail), PVC shows a low probability of response (Fig. 4b, c). Compared to upstream sensory neurons, PVC also responds with a lower magnitude and the response is more variable (Fig. 4b, c and Supplementary Fig. 12b, c). Perhaps not so surprisingly, PVC's response to subthreshold stimuli in the same modality can be sensitized for subsequent stimulation (Supplementary Fig. 12d). More 
interestingly, when pre-sensitized by cross-modality sub-threshold stimuli (i.e. chemical before mechanical stimulus, or vice versa), PVC shows similar sensitized responses (Fig. 4d, e, and Supplementary Movie 5,6). This sensitization is seen both in terms of the magnitude of the individual responses and the fraction of responding animals.

\section{Discussion}

For fundamental studies of mechanosensation, quantitative live imaging is necessary, and to perform screens based on mechanosensory phenotypes requires large sample sizes. Our microfluidic platform allows for studying mechanosensation in C. elegans quantitatively and conveniently, allowing for the delivery of a variety of types of mechanical stimuli to live animals while recording neuronal activity. Experimental preparation (mainly washing) can be accomplished for a batch of animals, so the limiting step is imaging (tens of seconds to minutes depending on the experiments). Experimental throughput using our streamlined microfluidic system can be as high as $\sim 100$ trials per hour; it is also straightforward to automate and run these systems in parallel to further improve throughput. In contrast, the conventional approach (gluing worms and stimulating with a micro stylus and micromanipulator) generally yields $\sim 10$ successful trials per day. The integration of hardware and software also allows for automated operations of imaging, stimulation, and quantitative analysis, further reducing potential human error and bias. This important improvement in throughput and standardization over conventional methods allowed us to conduct a novel drug screen based on neuronal dynamics due to mechanical stimuli. By using our system, we identified several candidates that strongly affect dynamics in mechanosensory neurons in a variety of ways. One can envision genetic screens performed in a similar manner to identify mechanosensory mutants. Many worm mechanosensory modalities, such as harsh touch and nose touch, involve multiple partiallyredundant cell types, making behavioral assays ineffective for finding genes affecting these processes. With simple integration of sorting mechanisms on chip ${ }^{36,42,43}$, it will be possible to conduct high-throughput forward screens for mutants affecting the responses of individual neurons, using a GECI-based assay. The genes identified in such screens should provide insight into the underlying mechanisms of mechanosensation, as well as find potential therapies for sensory-loss conditions such as deafness. 
Additionally, microfluidic incorporation of fluidic control can easily allow interrogation of other sensory modalities (e.g. olfaction) in combination with mechanosensation. We have shown that our platform is compatible with previous techniques for delivering chemical stimuli, enabling for the interrogation of integration of multimodal stimuli in the interneurons. This feature can greatly expand the repertoire of assay conditions to allow studies of sensory integration, arousal, habituation, and sensitization. For example, it has been previously shown that neural responses to sensory stimuli become more deterministic as information flows from sensory neurons to interneurons; behavioral responses, however, correlate more strongly with interneurons such as $\mathrm{PVC}^{33,44}$. We show here that PVC's response can be modulated, by prior sensory inputs, and that this modulation is cross-modal. This may point to an interesting ecologically relevant strategy for animal behavior, such that the reliability of the escape response depends both on the stimulus and on the current state of the circuit, as influenced by experience.

Because our platform employs a simple microfluidic device, it is easily adaptable to study biological systems of various sizes. Scaling the devices to be smaller can allow studies of mechanosensory neurons in worm larvae during development; scaling the devices larger can allow studies of the mechanosensation circuit during aging in C. elegans, as well as neurons and circuits in other model organisms such as zebrafish or fly larvae. Lastly, because the microfluidic chip allows unhindered optical access, integrations of optogenetic methods ${ }^{37,38,45-49}$ can also be straightforwardly carried out in this platform, thereby greatly expanding the repertoire of biological problems to be studied.

\section{Acknowledgments:}

The authors would like to thank K. Shen (HHMI/Stanford) and D. Kim (HHMI Janelia

252 Research Campus) for sharing reagents; GCaMP6 transgene constructs were provided by the 253 Genetically-Encoded Neuronal Indicator and Effector Project, Janelia Research Campus, 254 Howard Hughes Medical Institute. This work was supported in part by the U.S. NIH 255 (R01GM088333 and R01NS096581 to HL), the Wellcome Trust (WT103784MA to WRS), and the MRC (MC-A022-5PB91 to WRS). Some nematode strains used in this work were provided 
by the Caenorhabditis Genetic Center, which is funded by the NIH, National Center for Research

258 Resources and the International C. elegans Knockout Consortium.

259

260

261

262

263

264

265

266

267

268

269

270

271

272

273

274

275

276

277

278

279

280

281

282

283

284

285

286

287

\section{Methods:}

\section{$\underline{\text { Strains }}$}

C. elegans were maintained under standard conditions and fed OP50 bacteria ${ }^{50}$. The following strains were used in this study:

AQ 3236 ljIs142[mec-4::GCaMP6m::SL2TagRFP, unc-119] II; unc-119(ed3) III

TV17924 wyls5007[ser2prom3::GCaMP6, egl-17::mCherry] X

CX10979 kyEx2865[sra-6::GCaMP3, Pofm-1::GFP]

GT243 aEx2[pglr-1::GCaMP6(s), punc-122::GFP]

RW1596 stEx30[myo-3p::GFP + rol-6(su1006)]

To construct AQ3236, we used a single-copy insertion vector containing a GCaMP6M transgene codon-optimized for C. elegans, under the control of the mec-4 promoter (a gift from Doug Kim at HMMI Janelia Research Campus). Single-copy chromosomal integrations were obtained using the MosTic procedure ${ }^{51}$. Unless otherwise specified, all worms imaged in this study are adults.

\section{Chip Design and Fabrication}

The device consists of worm inlet/outlet, imaging channel (50 60 $\mu \mathrm{m}$ deep), and four sets of actuated PDMS membranes. Animals loosely fit in the channel, and are trapped (but not held) in the imaging area by two sets of actuated members. The width of actuated PDMS membrane is $150 \mu \mathrm{m}$, the distance between first and second sets of membrane is $200 \mu \mathrm{m}$ and second and third sets of membrane is $250 \mu \mathrm{m}$.

Since worms were not immobilized using drugs, animals' head or tail can move in the imaging channel of the microfluidic chip. This movement sometimes blurs images. To reduce the movement of head or tail part of worms, a three-step vertical tapering of the imaging channel was used to restrict the out-of-plane movement. The thickness of first and second layers was 15 $\mu \mathrm{m}$ and third layer was $20 \mu \mathrm{m}$ for the $50 \mu \mathrm{m}$ deep imaging channel; these layers were created by SU-8 2015 negative photoresist (MicroChem) using standard photolithographic techniques ${ }^{52}$.

To create the actuated PDMS structure to touch and trap worms, multi-layer soft lithography process ${ }^{53}$ was used. For the bottom flow layer of features, 23:1 PDMS was deposited via spin coating to create a thin layer. For the top control layer, 10:1 PDMS was directly poured 
onto a blank master, which does not have any features, to create a thick and mechanically rigid handle layer. Both layers were then placed into a $90^{\circ} \mathrm{C}$ oven for $25-30$ minutes until the control layer PDMS was rigid but sticky. After they were manually aligned, additional 10:1 PDMS was poured and cured for several hours to create a rigid handling layer for the device.

\section{Calcium Imaging}

All imaging experiments were performed on a Leica DMIRB inverted microscope using a 40x air objective (N.A. 0.75). Video sequences were captured using a Hamamatsu EM-CCD camera with $100 \mathrm{~ms}$ exposure time. Simultaneous two-color imaging was performed using a DV2 beamsplitter (Photometrics) containing a GFP/RFP filter set. Excitation light for fluorescent imaging was delivered through a projector system previously developed ${ }^{38}$. In experiments for the measurement of mechanosensory neuronal responses, stimuli were delivered $10 \mathrm{~s}$ after recording baseline activity of neurons. In experiment for the measurement of interneuronal recording, stimuli were delivered $30 \mathrm{~s}$ after recording baseline activity of neurons. Videos were recorded for 60-180 s following stimulus delivery.

\section{$\underline{\text { Data Analysis }}$}

Fluorescence intensities for each frame were extracted using customized neuron-tracking Matlab scripts (Supplementary Fig. 7). In strains where both GCaMP6 and RFP are expressed, the ratio between intensity values were computed $\left(R=\frac{I_{G_{-} R O I}}{I_{R_{-} R O I}}\right)$ in order to minimize movement artifacts. When only GCaMP was available, fluorescence values were computed by subtracting background intensity $\left(F=I_{G_{-} R O I}-I_{G_{B a c k}}\right)$. GCaMP and RFP intensities were measured as the mean pixel intensity of the 100 brightest pixels of a circular region of interest (ROI) of 10 pixel radius. Background intensities were subtracted to adjust for variations in lighting conditions, and were measured as the mean pixel intensity of an ROI in a background region (Supplementary Fig. 7). Calcium traces were computed as the change in $\mathrm{R}$ or $\mathrm{F}$ from the baseline value $\left(\frac{\Delta R}{R_{o}}=\right.$ $\left.\frac{R-R_{o}}{R_{o}}\right)$ or $\left(\frac{\Delta F}{F_{O}}=\frac{F-F_{o}}{F_{o}}\right)$. Baseline values were computed as the mean $\mathrm{R}$ or $\mathrm{F}$ prior to stimulus delivery. 
319 Drug screening

320 Worms were roughly synchronized by picking 20-25 L4 worms and allowing them to lay 321 eggs overnight before removing them from the plate. After two days at $20^{\circ} \mathrm{C}$, tightly age322 synchronized populations of worms were obtained by washing adults and L1s off of these plates 323 and then washing newly hatched L1s from these plates after an hour interval. The 84 compounds 324 of the Screen-Well Orphan library (ENZO) were used for the drug screening. 20-30 tightly325 synchronized L4 worms were placed on a 48-well plate (Greiner Bio-One) with $0.5 \mathrm{ml}$ OP50 326 bacteria (OD 5) for non-treated worms and both $0.495 \mathrm{ml} \mathrm{OP50}$ bacteria and $0.005 \mathrm{ml}(100 \mu \mathrm{M})$

327 drugs for drug-treated worms. After 24 hours, worms were imaged. Among 84 compounds in the 328 library, we tested the effect of 13 compounds on AVM neuronal responses at three different ages 329 (from day 1 adult to day 3 adult). These compounds were chosen randomly from the orphan 330 ligand library. 


\section{References}

3321 Corey, D. P. New TRP channels in hearing and mechanosensation. Neuron 39, 585-588 (2003).

3332 Kung, C. A possible unifying principle for mechanosensation. Nature 436, 647-654 (2005).

3343 Nicolson, T. The genetics of hearing and balance in zebrafish. Annu. Rev. Genet. 39, 9-22 (2005).

3354 Lumpkin, E. A. \& Caterina, M. J. Mechanisms of sensory transduction in the skin. Nature 445,

336 858-865 (2007).

3375 Chalfie, M. et al. The neural circuit for touch sensitivity in Caenorhabditis elegans. The Journal of 338 neuroscience 5, 956-964 (1985).

3396 Chalfie, M. \& Au, M. Genetic control of differentiation of the Caenorhabditis elegans touch 340 receptor neurons. Science 243, 1027-1033 (1989).

3417 Hong, K. \& Driscoll, M. A transmembrane domain of the putative channel subunit MEC-4 influences mechanotransduction and neurodegeneration in C. elegans. (1994). Huang, M. \& Chalfie, M. Gene interactions affecting mechanosensory transduction in Caenorhabditis elegans. Nature 367, 467-470 (1994).

9 Corey, D. P. \& Garcia-Anoveros, J. Mechanosensation and the DEG/ENaC ion channels. Science 273, 323 (1996).

10 Tavernarakis, N., Shreffler, W., Wang, S. \& Driscoll, M. unc-8, a DEG/ENaC family member, encodes a subunit of a candidate mechanically gated channel that modulates $C$. elegans locomotion. Neuron 18, 107-119 (1997). functions and subcellular localization in C. elegans neurons. Neuron 35, 307-318 (2002). Goodman, M. B. et al. MEC-2 regulates C. elegans DEG/ENaC channels needed for mechanosensation. Nature 415, 1039-1042 (2002).

13 O'Hagan, R., Chalfie, M. \& Goodman, M. B. The MEC-4 DEG/ENaC channel of Caenorhabditis elegans touch receptor neurons transduces mechanical signals. Nature neuroscience 8, 43-50 (2005).

Kindt, K. S. et al. Caenorhabditis elegans TRPA-1 functions in mechanosensation. Nature neuroscience 10, 568-577 (2007). Chatzigeorgiou, M. et al. Specific roles for DEG/ENaC and TRP channels in touch and thermosensation in C. elegans nociceptors. Nature neuroscience 13, 861-868 (2010). Delmas, P. \& Coste, B. Mechano-gated ion channels in sensory systems. Cell 155, 278-284 (2013). elegans. Journal of Comparative Neurology 163, 215-226 (1975). Caenorhabditis elegans. Developmental biology 82, 358-370 (1981). Way, J. C. \& Chalfie, M. The mec-3 gene of Caenorhabditis elegans requires its own product for maintained expression and is expressed in three neuronal cell types. Genes \& development $\mathbf{3}$, 1823-1833 (1989). role for the MEC-4 channel in the process of gentle touch sensation. Neuron 39, 1005-1017 (2003). touch. Proceedings of the National Academy of Sciences 112, E6955-E6963 (2015).

Whitesides, G. M. The origins and the future of microfluidics. Nature 442, 368-373 (2006). San-Miguel, A. \& Lu, H. Microfluidics as a tool for C. elegans research. (2005).

Ryu, W. S. \& Samuel, A. D. Thermotaxis in Caenorhabditis elegans analyzed by measuring responses to defined thermal stimuli. The Journal of neuroscience 22, 5727-5733 (2002). 
Chronis, N., Zimmer, M. \& Bargmann, C. I. Microfluidics for in vivo imaging of neuronal and behavioral activity in Caenorhabditis elegans. Nature methods 4, 727-731 (2007).

26 Chalasani, S. H. et al. Dissecting a circuit for olfactory behaviour in Caenorhabditis elegans. Nature 450, 63-70 (2007).

27 Kuhara, A. et al. Temperature sensing by an olfactory neuron in a circuit controlling behavior of C. elegans. Science 320, 803-807 (2008).

28 Guo, Z. V., Hart, A. C. \& Ramanathan, S. Optical interrogation of neural circuits in Caenorhabditis elegans. Nature methods 6, 891-896 (2009).

29 Macosko, E. Z. et al. A hub-and-spoke circuit drives pheromone attraction and social behaviour in C. elegans. Nature 458, 1171-1175 (2009).

$30 \mathrm{Ha}, \mathrm{H} .-\mathrm{i}$. et al. Functional organization of a neural network for aversive olfactory learning in Caenorhabditis elegans. Neuron 68, 1173-1186 (2010).

31 Albrecht, D. R. \& Bargmann, C. I. High-content behavioral analysis of Caenorhabditis elegans in precise spatiotemporal chemical environments. Nature methods 8, 599-605 (2011).

32 Kato, S., Xu, Y., Cho, C. E., Abbott, L. \& Bargmann, C. I. Temporal responses of C. elegans chemosensory neurons are preserved in behavioral dynamics. Neuron 81, 616-628 (2014).

33 Luo, L. et al. Dynamic encoding of perception, memory, and movement in a C. elegans chemotaxis circuit. Neuron 82, 1115-1128 (2014).

34 Chen, T.-W. et al. Ultrasensitive fluorescent proteins for imaging neuronal activity. Nature 499, 295-300 (2013).

35 Li, W., Kang, L., Piggott, B. J., Feng, Z. \& Xu, X. S. The neural circuits and sensory channels mediating harsh touch sensation in C. elegans. Nature communications 2, 315 (2011).

36 Crane, M. M., Chung, K., Stirman, J. \& Lu, H. Microfluidics-enabled phenotyping, imaging, and screening of multicellular organisms. Lab on a Chip 10, 1509-1517 (2010).

37 Leifer, A. M., Fang-Yen, C., Gershow, M., Alkema, M. J. \& Samuel, A. D. Optogenetic manipulation of neural activity in freely moving Caenorhabditis elegans. Nature methods 8, 147152 (2011).

38 Stirman, J. N. et al. Real-time multimodal optical control of neurons and muscles in freely behaving Caenorhabditis elegans. Nature methods 8, 153-158 (2011).

39 Hilliard, M. A., Bargmann, C. I. \& Bazzicalupo, P. C. elegans responds to chemical repellents by integrating sensory inputs from the head and the tail. Current Biology 12, 730-734 (2002).

40 White, J. G., Southgate, E., Thomson, J. N. \& Brenner, S. The structure of the nervous system of the nematode Caenorhabditis elegans. Philos Trans R Soc Lond B Biol Sci 314, 1-340 (1986).

41 Wicks, S. R. \& Rankin, C. H. Integration of mechanosensory stimuli in Caenorhabditis elegans. The Journal of neuroscience 15, 2434-2444 (1995).

42 Chung, K., Crane, M. M. \& Lu, H. Automated on-chip rapid microscopy, phenotyping and sorting of C. elegans. Nature methods 5, 637-643 (2008).

43 Crane, M. M., Chung, K. \& Lu, H. Computer-enhanced high-throughput genetic screens of C. elegans in a microfluidic system. Lab Chip 9, 38-40 (2009).

44 Gordus, A., Pokala, N., Levy, S., Flavell, S. W. \& Bargmann, C. I. Feedback from network states generates variability in a probabilistic olfactory circuit. Cell 161, 215-227 (2015).

45 Boyden, E. S., Zhang, F., Bamberg, E., Nagel, G. \& Deisseroth, K. Millisecond-timescale, genetically targeted optical control of neural activity. Nature neuroscience 8, 1263-1268 (2005).

46 Zhang, F. et al. Multimodal fast optical interrogation of neural circuitry. Nature 446, 633-639 (2007).

47 Airan, R. D., Thompson, K. R., Fenno, L. E., Bernstein, H. \& Deisseroth, K. Temporally precise in vivo control of intracellular signalling. Nature 458, 1025-1029 (2009).

48 Deisseroth, K. Optogenetics. Nature Methods 8, 26-29, doi:10.1038/nmeth.f.324 (2011). 
49 Larsch, J. et al. A Circuit for Gradient Climbing in C. elegans Chemotaxis. Cell Rep 12, 1748-1760 (2015).

$42950 \quad$ Brenner, S. The genetics of Caenorhabditis elegans. Genetics 77, 71-94 (1974).

$43051 \quad$ Frøkjær-Jensen, C. et al. Single-copy insertion of transgenes in Caenorhabditis elegans. Nature genetics 40, 1375-1383 (2008).

52 del Campo, A. \& Greiner, C. SU-8: a photoresist for high-aspect-ratio and 3D submicron lithography. Journal of Micromechanics and Microengineering 17, R81 (2007).

53 Unger, M. A., Chou, H.-P., Thorsen, T., Scherer, A. \& Quake, S. R. Monolithic microfabricated valves and pumps by multilayer soft lithography. Science 288, 113-116 (2000). 
Figure 1: Microfluidic platform can robustly deliver mechanical stimulus and allow imaging of calcium responses in C. elegans mechanoreceptor neurons.

a) The device employs multiple sets of actuated structures: valves to trap animals in a reproducible position, and two sets of actuation valves used to deliver mechanical stimuli to the anterior and posterior regions of the body.

b) Sample frames from an activated neuron show changes in fluorescence due to mechanical stimulus. Because animals are not fully immobilized, and neurons of interest move during recordings due to the mechanical stimulus and behavioral responses, a tracking algorithm (Supplementary Fig. 3) was developed in order to automatically record the GCaMP and RFP intensities of traces from individual trials.

c) Timeline of on-chip mechano-stimulation and functional imaging of neurons. The loading and unloading of each worm requires only a few seconds. Each animal is given a waiting period to acclimate to the environment before being stimulated and imaged. Each trial is performed by recording video to track neuronal dynamic responses and applying mechanical stimuli.

d) Schematics of the mechanoreceptor neurons in this study: six gentle touch neurons -AVM, ALML/R, PVM, and PLML/R - and harsh touch PVDL/R neurons.

e - j) Responses of the C. elegans gentle touch and harsh touch neurons to mechanical stimuli. Average traces of GCaMP6 signals in e) ALM soma to 1s stimulus ( $\mathrm{n}=16), \mathbf{f})$ ALM process to $2 \mathrm{~s}$ stimulus ( $\mathrm{n}=7)$, g) PVD soma ( $\mathrm{n}=9,55 \mathrm{psi})$, h) PVM soma $(\mathrm{n}=17)$, i) PLM soma ( $\mathrm{n}=9)$, and $\mathbf{j})$ PVD process to $1 \mathrm{~s}$ stimulus $(\mathrm{n}=5)$ at $45 \mathrm{psi}$. Error bars represent SEM. stimulus was delivered to the appropriate regions of animals in our system. k) The activity of AVM responses to $1 \mathrm{~s}$ anterior but not posterior stimuli (anterior: $\mathrm{n}=10$, posterior: $\mathrm{n}=5$ ) at 45 psi. touch neuron, PVD, (2s stimulation, anterior: $n=9$, posterior: $n=3)$ respond to both anterior and

464 stimuli than anterior stimuli. Error bars represent SEM. Orange denotes anterior touch and green 465 denotes posterior touch. 
bioRxiv preprint doi: https://doi.org/10.1101/107318; this version posted February 10,2017 . The copyright holder for this preprint (which was not certified by peer review) is the author/funder, who has granted bioRxiv a license to display the preprint in perpetuity. It is made available under aCC-BY-NC-ND 4.0 International license.

466 For panels (e-m), arrow thickness indicates stimulation duration. 1s and $2 \mathrm{~s}$ stimulations are 467 represented by thin and thick arrows, respectively. 
Figure 2: The microfluidic platform delivers mechanical stimuli emulating both gentle and harsh touch by varying the magnitude of applied pressure and duration of the stimuli.

a - b) Average traces of GCaMP6 signal in AVM neuron in response to diverse pressures and stimulus durations. a) Applied 1s stimulation with diverse pressures (25 psi: n=11, 35 psi: n=25, 40 psi: $n=8,45$ psi: $n=27)$.

b) Applied 40 psi stimulation with diverse stimulus durations (1s: $\mathrm{n}=8,2 \mathrm{~s}: \mathrm{n}=10,5 \mathrm{~s}: \mathrm{n}=10)$.

c - d) Maximum responses of calcium transients correlate with c) the applied pressure (2s stimulus, 35 to $45 \mathrm{psi}$ ) and d) the duration of stimuli (1 to $5 \mathrm{~s}$ stimuli, $40 \mathrm{psi}$ ). Error bars represent SEM.

e) Average calcium responses of mec-4(e1611) mutants in AVM neuron to diverse pressures with 1s stimulus (25 psi: $n=18,35$ psi: $n=10,40$ psi: $n=9,45$ psi: $n=10$ ).

f) Maximum responses of calcium responses of wild-type and mec-4 mutant animals (MannWhitney Test, $* \mathrm{p}<0.05, * * \mathrm{p}<0.01, * * * \mathrm{p}<0.001, * * * * \mathrm{p}<0.0001)$.

g - h) Average traces of GCaMP6 signal in PVD neuron in response to diverse pressures and stimulus durations. g) Applied 1s stimulation with diverse pressures (45 psi: n=9, 50 psi: n=6, 55 psi: n=9). h) Applied 45 psi stimulation with diverse stimulus durations (1s: n=9, 2s: n=4, 5s: $\mathrm{n}=6$ ).

i) Quantitative responses of AVM and PVD under different stimulation conditions. Both gentle touch AVM neurons and harsh touch sensing PVD neurons can be stimulated using this platform when using the right parameter regime. Each column refers to the applied pressure magnitude and each row refers to the applied durations of stimulation. For each data point, the circle size indicates the max response value from 0 to 3.0 and the rectangle size indicates response fraction from 0 to 1 . Response fraction is defined as the percentage of traces that show a max response 492 value of higher than 0.5 .

j-m) Delivery of precisely repeated stimuli in AVM. j, l) When worms are exposed to 1s stimuli with short inter-stimulus intervals (1s), the neurons exhibited an incremental increase in response magnitude up to the second stimulus, and a reduced response in later stimuli $(\mathrm{n}=19)$. $\mathbf{k}, \mathbf{m})$ In 496 contrast, when exposed to $2 \mathrm{~s}$ stimuli with long inter-stimulus intervals (3 min), the response magnitude was reduced after each stimulus $(n=10)$. Error bars represent SEM. 
Figure 3: The microfluidic platform enables screens to examine compounds that may affect neuronal responses to mechanical stimuli.

501

502

503

504

505

506

507

508

509

510

511

512

513

514

515

516

517

518

519

520

521

522

a) Experimental procedure for the drug screen performed. Synchronized L1 worms are grown in NGM plates to the L4 stage and then deposited in a 48-well plate. Drug treated worms are cultured with $0.5 \mathrm{ml} \mathrm{OP50} \mathrm{E.} \mathrm{coli} \mathrm{bacteria} \mathrm{(OD} \mathrm{5)} \mathrm{and} \mathrm{100 \mu M} \mathrm{drugs.} \mathrm{Control} \mathrm{worms} \mathrm{are} \mathrm{cultured}$

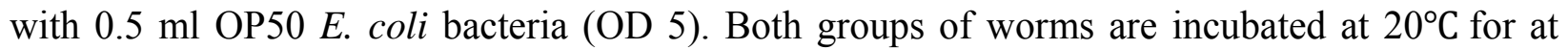
least $24 \mathrm{~h}$. Subsequently, AVM responses to 1s stimulus were measured on-chip.

b) Three metrics measured from individual calcium dynamic traces: maximum response, delay time (time between the end of the stimulus and the arrival of maximum response), and half-life (time it takes the response to decay to half of the maximum).

c) Fraction of animal responses upon compound treatment. Several compounds produced a lowered fraction of responding animals, while a few slightly increased the response fraction.

d - f) Box plots show how compounds affect specific parameters of neuronal response upon mechanical stimulation. Quantification of each response was normalized to that of the control group from the same day (day 1 adult to day 3 adult). d) D-Lysine (\#4), D-Alanine (\#5), and DArginine (\#8) were shown to reduce maximum response, e) D-Alanine (\#5) and D-Arginine (\#8) were shown to increase the time to peak, and f) D-Isoluecine (\#3) was shown to decrease the decay half-life. In contrast, $\beta$-Alanine (\#6) was shown to increase the half-life. (Kruskal-Wallis Test, $* \mathrm{p}<0.05, * * \mathrm{p}<0.01, * * * \mathrm{p}<0.001, * * * * \mathrm{p}<0.0001)$

g - h) Average traces of GCaMP6 in AVM neuron for drug treated worms that cause significant differences from untreated worms in responses to $1 \mathrm{~s}$ mechanical stimulus. g) Day 1 adult worms (Control Day 1: $n=53$, D-Isoleucine: $n=10$, D-Lysine: $n=10)$, h) Day 2 adult worms (Control Day 2: $n=53$, D-Alanine: $n=15$, $\beta$-Alanine: $n=14$, D-Arginine: $n=13$ ). Error bar represent SEM. 
523 Figure 4: Sensitization of the PVC interneuron responses.

524 a) Simplified circuit diagram showing three sensory neurons connecting PVC to forward locomotion behavior (values and arrow thickness indicate number of synapses).

526 b - c) Responses of PVC interneuron to a single pulse of stimulation. Averages are plotted on the 527 top graph. Error bars represent SEM. Bottom graphs represent individual traces. c) PVC calcium 528 responses to 30 s $0.1 \%$ SDS stimuli on tail $(n=23)$. d) PVC calcium responses to $1 \mathrm{~s}$ weak 529 mechanical stimuli at 20 psi on posterior region $(n=18)$. In individual traces for outliers, if the 530 value of calcium transient is greater than 4 or less than 0 , it would be equal to 4 or 0 , respectively 531 (bottom).

532 d - e) Sensitized PVC interneuron responses. e) Applying 5s 0.1\% SDS stimuli enhances the 533 responses of PVC interneuron to $1 \mathrm{~s}$ weak mechanical stimuli at $20 \mathrm{psi}(\mathrm{n}=31)$. Averaged calcium responses (top) and individual traces (bottom) f) Applying 1s weak mechanical stimuli at 20 psi

535 enhances the responses of PVC interneuron to 30s $0.1 \%$ SDS stimuli $(n=24)$. Averaged calcium 536 responses (top) and individual traces (bottom). Error bars represent SEM (top). In individual 537 traces for outliers, if the value of calcium transient is greater than 4 or less than 0 , it would be 538 equal to 4 or 0 , respectively (bottom).

539 f) Quantified maximum responses of calcium transients to either chemical (left column) or 540 mechanical stimuli (right column). Data points in control groups represent maximum responses 541 to either single chemical or mechanical stimuli. Sensitization of PVC interneuron responses is 542 produced by applying prior weak mechanical stimuli at 20 psi or $5 \mathrm{~s} 0.1 \%$ SDS chemical stimuli 543 (Mann-Whitney Test, * $\mathrm{p}<0.05, * * \mathrm{p}<0.01$ ). 
Supplementary Figure 1: Overview of microfluidic device design and dimensions. The device is composed of the channel for worms (50-60 $\mu \mathrm{m}$ deep and wide, which allow the animals to fit loosely inside), two sets of actuated membrane, and two sets of trapping valve. The width of the both of actuated PDMS membrane and trapping valve is $150 \mu \mathrm{m}$, the distance between first and second sets of membrane is $200 \mu \mathrm{m}$ and second and third sets of membrane is $250 \mu \mathrm{m}$.

Supplementary Figure 2: Displacement of the actuated membrane by applying pressure ( $\mathrm{n}=4$ worms). It is important to note that the measurements were taken by images from transgenic worms expressing GFP along body-wall muscle (stEx30[myo-3p::GFP + rol-6(su1006)]). Rsquare value is 0.9814 .

Supplementary Figure 3: Neuron Tracking Algorithm. In order to extract fluorescence intensities throughout recordings, a neuron tracking algorithm was developed. This was necessary because worms are not fully immobilized in the device, and mechanical stimuli often caused the neuron of interest to move within the field-of-view. a) Overall schematic of the neuron tracking algorithm. For each frame $i$, raw images are processed through a blob filter (Laplace of Gaussian filter) to improve contrast and facilitate segmentation. Blob filtered images are segmented by applying an empirically determined threshold. The neuron of interest is identified by the user in the first frame, and by distance to the neuron in the previous frame. Lastly, once the neuron is detected for each frame, intensity values are extracted (Green ROI, Red ROI, Green Background, and Red Background). b) Example of algorithm procedure.

Supplementary Figure 4: a) Average traces of GCaMP6 signal in AVM neuron in response to diverse pressures and stimulus durations (i-iii: 35 psi, iv-vi: 40 psi, vii-ix: 45 psi / i, iv, vii: 1s stimulus, ii, v, viii: $2 \mathrm{~s}$ stimulus, iii, vi, ix: 5 s stimulus, sample size i: $n=25$, ii: $n=10$, iii: $n=8$, iv: $n=8, \mathbf{v}: n=10$, vi: $n=10$, vii: $n=27$, viii: $n=6$, ix: $n=10$ ). Error bars represent SEM. b) Average traces of GCaMP6 signal in PVD neuron in response to diverse pressures and stimulus durations (i-iii: 45 psi, iv-vi: 50 psi, vii-ix: 55 psi / i, iv, vii: 1s stimulus, ii, v, viii: 2 s stimulus, iii, vi, ix: 5s 
575

576

577

578

579

580

581

582

583

584

585

586

587

588

589

590

591

592

593

594

595

596

597

598

599

600

601

602

stimulus, sample size i: $n=9$, ii: $n=4$, iii: $n=6$, iv: $n=6, \mathbf{v}: n=9$, vi: $n=10$, vii: $n=9$, viii: $n=10$, ix: $\mathrm{n}=10$ ). Error bar represent SEM.

Supplementary Figure 5: AVM cell body responses to various stimuli with low pressures and durations a) 30 psi and $1 \mathrm{~s}(\mathrm{n}=4)$, b) 30 psi and $0.2 \mathrm{~s}(\mathrm{n}=10)$, c) 15 psi and $0.2 \mathrm{~s}(\mathrm{n}=5)$. AVM response is reduced when using lower pressures (comparing a to Fig. 2a, and a to c). Response is also attenuated when using shorter durations (comparing a to b). Error bars represent SEM.

Supplementary Figure 6: PLM cell body responses to various stimulus durations $(1 \mathrm{~s}: \mathrm{n}=9,2 \mathrm{~s}$ : $n=4,5 s: n=4)$. Similar to those of AVM, maximum responses in PLM were proportional to the stimulus duration.

Supplementary Figure 7: AVM cell body response to delivery of repeated stimuli with long durations $(5 \mathrm{~s}, \mathrm{n}=5)$. Similar to Figure 21, traces showed incremental increases in the first few stimuli, and showed a decreased response in later stimuli.

Supplementary Figure 8: Individual (gray) and average traces (blue) for AVM response in untreated animals for different control groups for drug screen. a) Day $1(n=53), \mathbf{b})$ Day $2(n=53)$, c) Day $3(n=35)$ adult worms.

Supplementary Figure 9: Average traces for AVM response in drug-treated animals that do not show a significant difference from the control groups. a) Day 1 adult worms (Control Day 1: n=53, D-Glutamic acid: $n=10$, D-Serine: $n=12$ ), b) Day 2 adult worms (Control Day 2: $n=53, \gamma$ D-Glutamylglycine: $n=10$, D-Asparagine: $n=4$ ), c) Day 3 adult worms (Control Day 3: n=35, 1,1'Ethylidene-bis(L-tryptophan): n=10, D-Cysteine: $\mathrm{n}=10$, D-Glutamine: $\mathrm{n}=11$, D-Histidine: $\mathrm{n}=13$ ).

Supplementary Figure 10: Overview of microfluidic device for the delivery of multimodal stimuli. The device is composed of a channel for worms (Inlet and Flush channel), two sets of 
603 actuated membrane for mechanical stimuli (Touching valve 1,2), one set of trapping valve

604 (Loading valve), two inlets for chemical stimuli (Buffer and Stimulus), and outlet.

605

606 Supplementary Figure 11: Average traces of GCaMP3 signal in ASH neuron in response to 30s

$6070.1 \%$ SDS stimuli $(\mathrm{n}=13)$. Stimuli were delivered $30 \mathrm{~s}$ after recording baseline activity of 608 neurons. Error bars represent SEM.

609

610 Supplementary Figure 12: a) Neural wiring diagram showing five sensory neurons in a circuit

611 linking PVC to forward behavior, and the number of direct synapses between each pair of

612 neurons (arrow thickness indicates number of synapses). b-c) The activity of PVC responses to

613 localized strong mechanical stimuli: b) $1 \mathrm{~s}$ anterior stimuli $(\mathrm{n}=5)$ and c) $1 \mathrm{~s}$ posterior stimuli

$614(\mathrm{n}=18)$ at $45 \mathrm{psi}$. d) Applying prior 5s $0.1 \%$ SDS stimuli enhances the responses of PVC

615 interneuron to next 30 s $0.1 \%$ SDS stimuli $(n=13)$. e) The activity of PVC responses to buffer to

616 buffer changes $(\mathrm{n}=10)$. Error bars represents SEM.

617

618 
619 Supplementary Table 1: The 13 compounds of the orphan library were used for the drug screen

620 (Fig. 3). Sample size is the total number of tested worms and if the value of maximum responses

621 is larger than 0.5 , it is counted as a responding worm.

622

623 
Supplementary Movie 1: Calcium dynamics of AVM neuron to 1s anterior stimulation at 45psi. Stimulus was delivered 10s after recording baseline of neuronal activity. The transgenic animal shown here expresses GCaMP6 and RFP in AVM neuron (ljIs142[mec4::GCaMP6m::SL2TagRFP, unc-119] II; unc-119(ed3) III). Left panel shows green fluorescence from GCaMP6m (left) and red fluorescence from RFP (right) in false colors. White boxes indicate location of AVM neuron and shows how algorithm tracks the neuron. Right graph shows the quantitative calcium trance and red circle indicates the current time point of video. Stimulus occurs at 10s (red dash line). 1x playback.

Supplementary Movie 2: Calcium dynamics of PLM neuron to 1s posterior stimulation at 45psi. Stimulus was delivered 10s after recording baseline of neuronal activity. The transgenic animal shown here expresses GCaMP6 and RFP in PLM neuron (ljIs142[mec4::GCaMP6m::SL2TagRFP, unc-119] II; unc-119(ed3) III). Left panel shows green fluorescence from GCaMP6m (left) and red fluorescence from RFP (right) in false colors. White boxes indicate location of PLM neuron and shows how algorithm tracks the neuron. Right graph shows the quantitative calcium trance and red circle indicates the current time point of video. Stimulus occurs at 10s (red dash line). 1x playback.

Supplementary Movie 3: Calcium dynamics of PVM neuron to 1s posterior stimulation at 45psi. Stimulus was delivered 10s after recording baseline of neuronal activity. The transgenic animal shown here expresses GCaMP6 and RFP in PVM neuron (ljIs142[mec4::GCaMP6m::SL2TagRFP, unc-119] II; unc-119(ed3) III). Left panel shows green fluorescence from GCaMP6m (left) and red fluorescence from RFP (right) in false colors. White boxes indicate location of PVM neuron and shows how algorithm tracks the neuron. Right graph shows the quantitative calcium trance and red circle indicates the current time point of video. Stimulus occurs at 10s (red dash line). 1x playback.

Supplementary Movie 4: Calcium dynamics of PVD neuron to 1s posterior stimulation at 45psi. Stimulus was delivered 10s after recording baseline of neuronal activity. The transgenic animal shown here expresses GCaMP6 in PVD neuron (wyls5007[ser2prom3::GCaMP6, egl- 
17::mCherry] X). Left panel shows green fluorescence from GCaMP6 in false color. A white box indicates location of PVD neuron and shows how algorithm tracks the neuron. Right graph shows the quantitative calcium trance and red circle indicates the current time point of video. Stimulus occurs at 10s (red dash line). 5x playback.

Supplementary Movie 5: Calcium dynamics of PVC interneuron. Applying 5s 0.1\% SDS chemical stimulus at 30s (red dash lines on the right panel) and then 1s weak mechanical stimuli at 65s (blue dash lines on the right panel). The transgenic animal shown here expresses GCaMP6 in PVC interneuron (aEx2[pglr-1::GCaMP6(s), punc-122::GFP]). Left panel shows green fluorescence from GCaMP6 in false color. A white box indicates location of PVC interneuron and shows how algorithm tracks the neuron. Right graph shows the quantitative calcium trance and red circle indicates the current time point of video. Stimulus occurs at 10s (red dash line). 5x playback.

Supplementary Movie 6: Calcium dynamics of PVC interneuron. Applying 1s weak mechanical stimulus at 30s (blue dash lines on the right panel) and then $30 \mathrm{~s} 0.1 \%$ SDS chemical stimulus at 60s (red dash lines on the right panel). The transgenic animal shown here expresses GCaMP6 in PVC interneuron (aEx2[pglr-1::GCaMP6(s), punc-122::GFP]). Left panel shows green

672 fluorescence from GCaMP6 in false color. A white box indicates location of PVC interneuron 673 and shows how algorithm tracks the neuron. Right graph shows the quantitative calcium trance 674 and red circle indicates the current time point of video. Stimulus occurs at 10s (red dash line). 5x 675 playback.

\section{Methods References}

679

68054 Brenner, S. The genetics of Caenorhabditis elegans. Genetics 77, 71-94 (1974).

$68155 \quad$ Frøkjær-Jensen, C. et al. Single-copy insertion of transgenes in Caenorhabditis elegans. Nature 682 genetics 40, 1375-1383 (2008).

68356 del Campo, A. \& Greiner, C. SU-8: a photoresist for high-aspect-ratio and 3D submicron 684 lithography. Journal of Micromechanics and Microengineering 17, R81 (2007). 
bioRxiv preprint doi: https://doi.org/10.1101/107318; this version posted February 10,2017 . The copyright holder for this preprint (which was not certified by peer review) is the author/funder, who has granted bioRxiv a license to display the preprint in perpetuity. It is made available under aCC-BY-NC-ND 4.0 International license.

685

686

687
57 Unger, M. A., Chou, H.-P., Thorsen, T., Scherer, A. \& Quake, S. R. Monolithic microfabricated valves and pumps by multilayer soft lithography. Science 288, 113-116 (2000). 
a

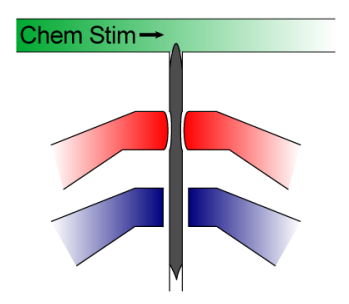

Anterior Touch /

Chem Stimulus on Worm Nose b

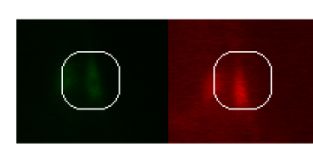

Pressure Off

Pressure On for Worm Touching

Worm

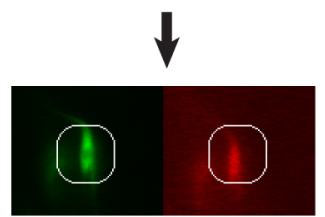

Before the stimulation

C
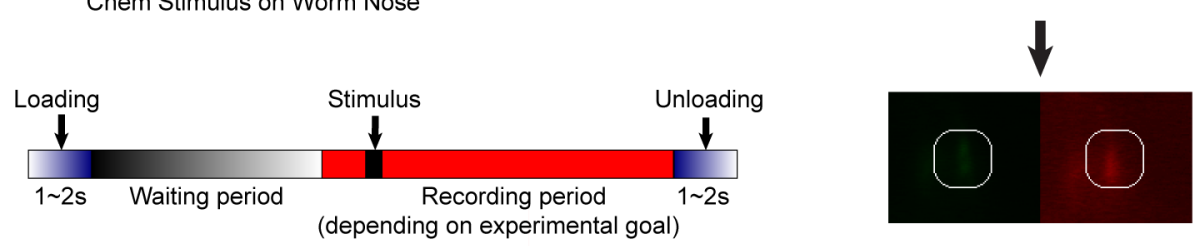

Right after the stimulation

d
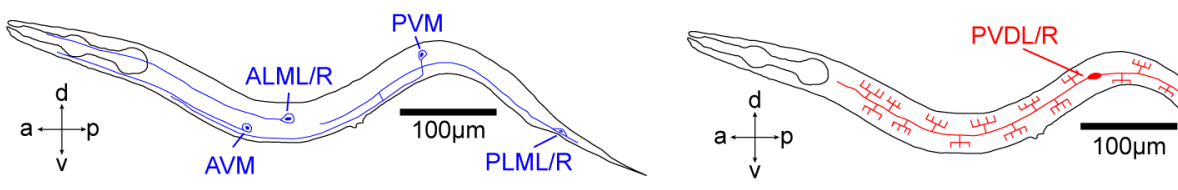

While after the stimulation

e

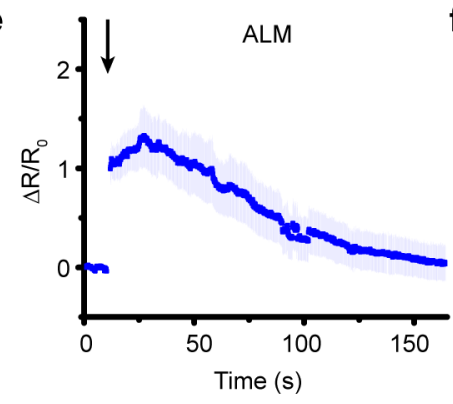

h

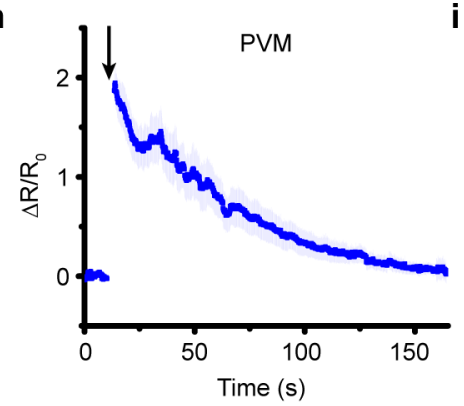

k

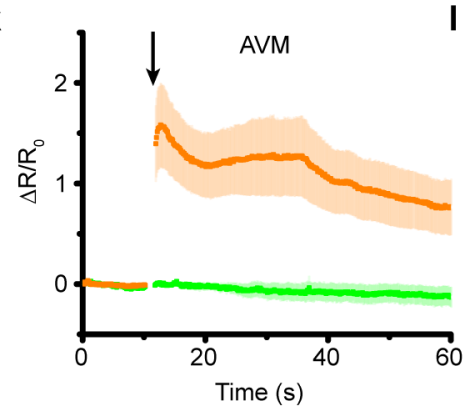

i

$\sqrt{10}+x^{2}$ f $\downarrow$ ALM

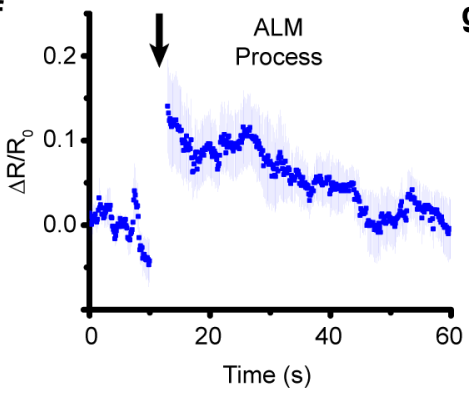

$9 \quad$ PVD

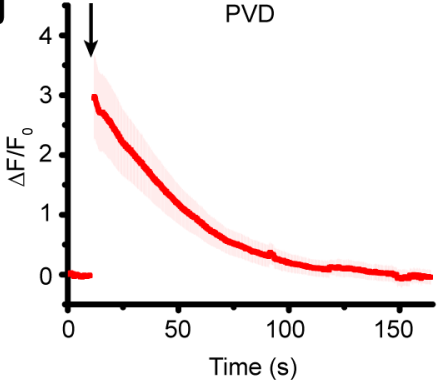

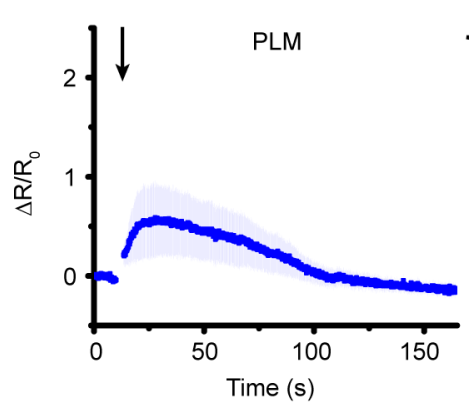
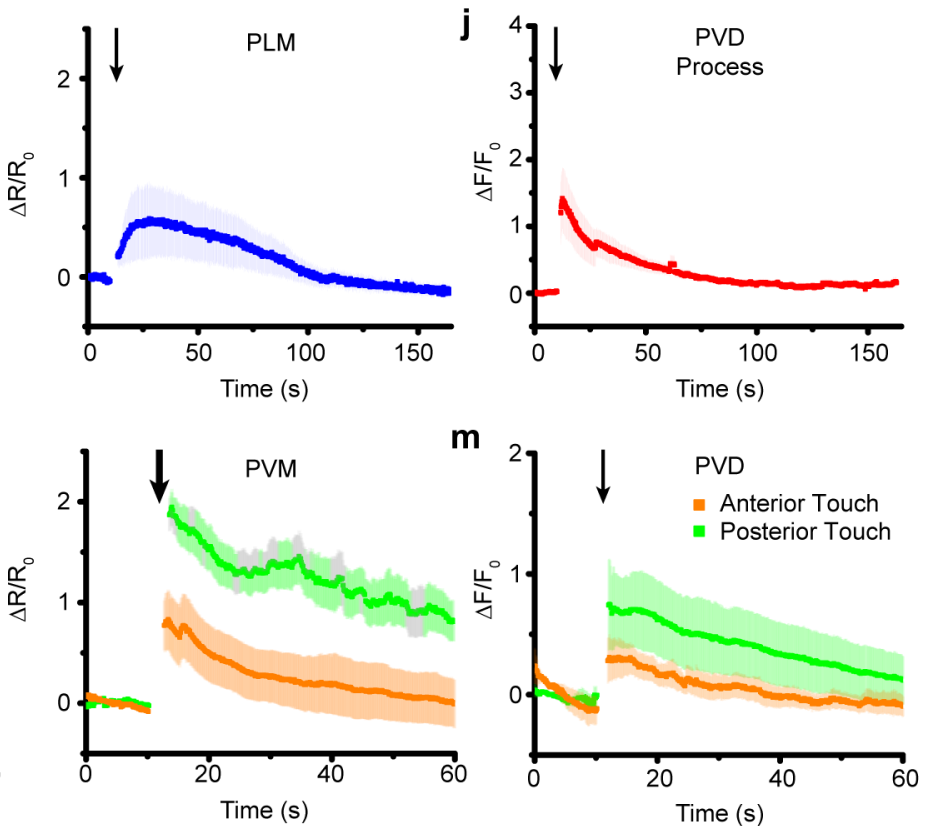

Cho et al., Figure 1. 
bioRxiv preprint doi: https://doi.org/10.1101/107318; this version posted February 10, 2017. The copyright holder for this preprint (which was not certified by peer review) is the author/funder, who has granted bioRxiv a license to display the preprint in perpetuity. It is made available

a

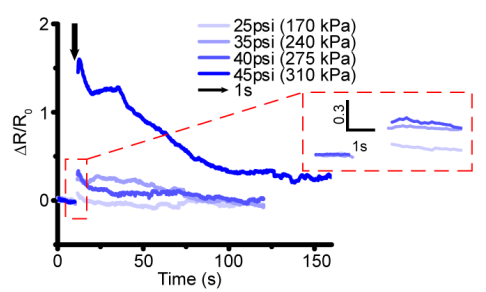

e

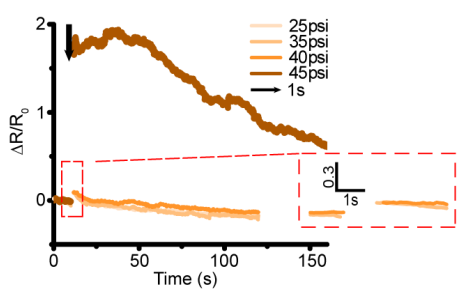

b

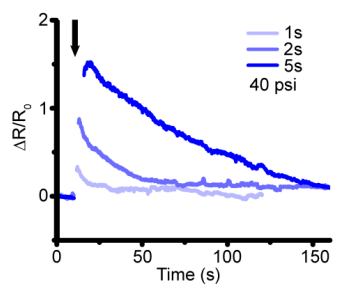

f

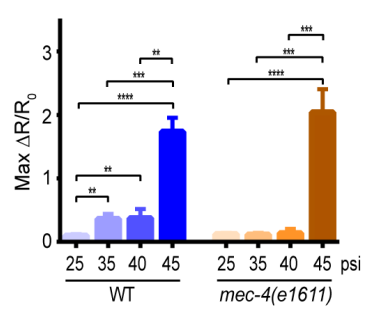

c

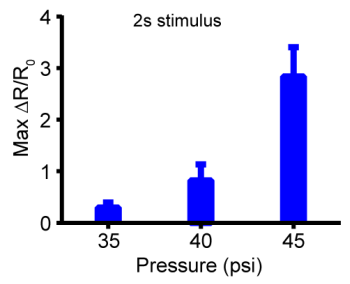

g

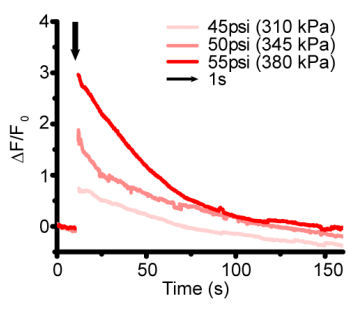

d

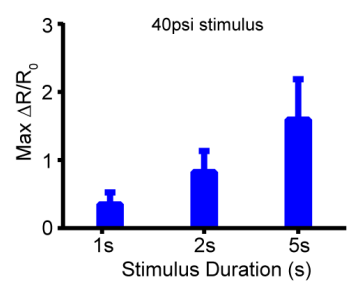

h

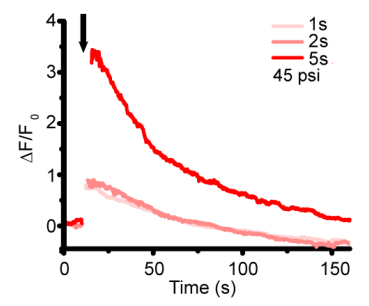

i
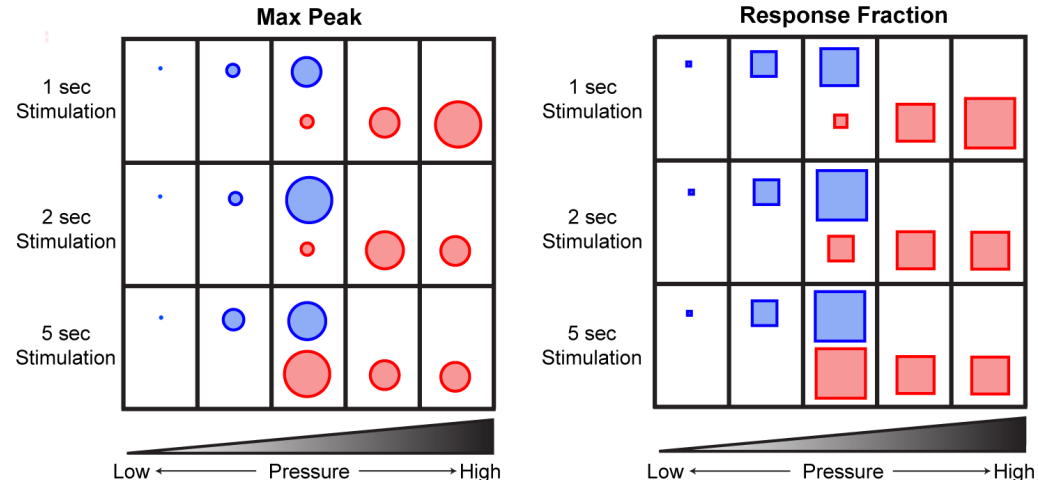

Legend:

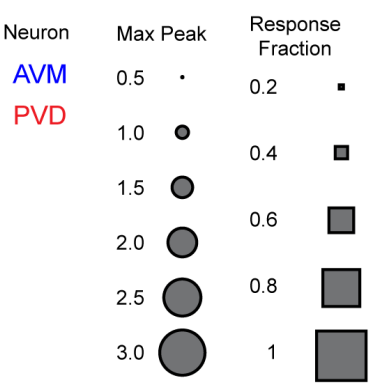

j

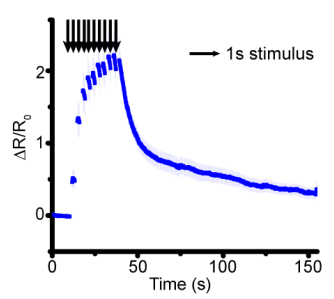

k

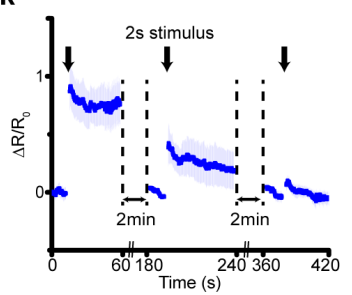

I

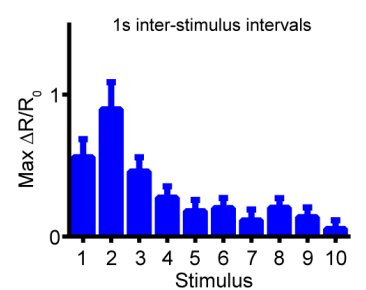

m

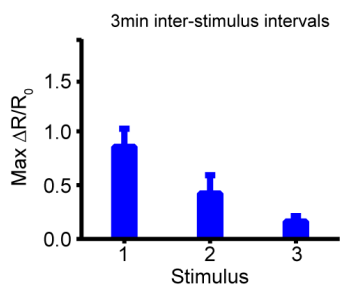

Cho et al., Figure 2. 
a

Synchronized
L1 Larvae

Drug Treated worms

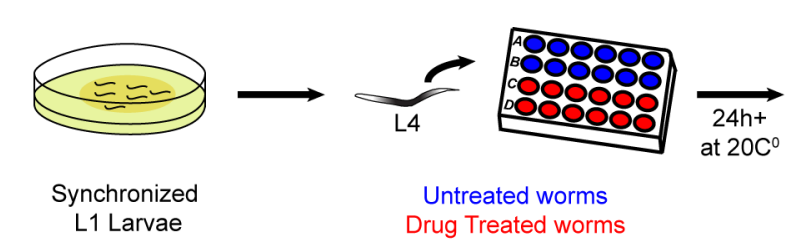

b

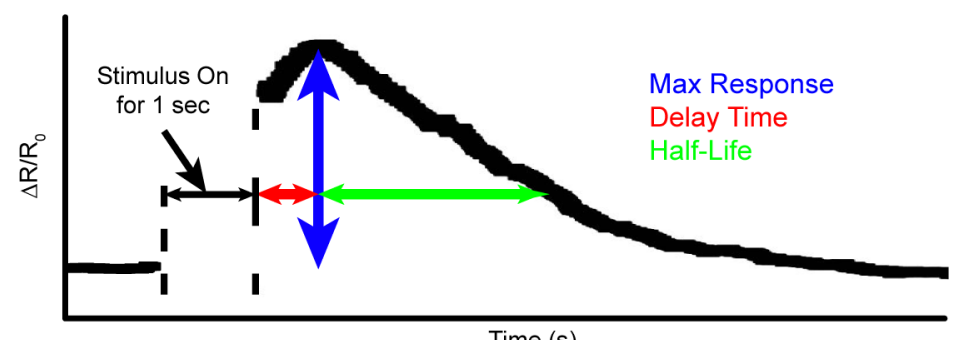

Time (s)

d
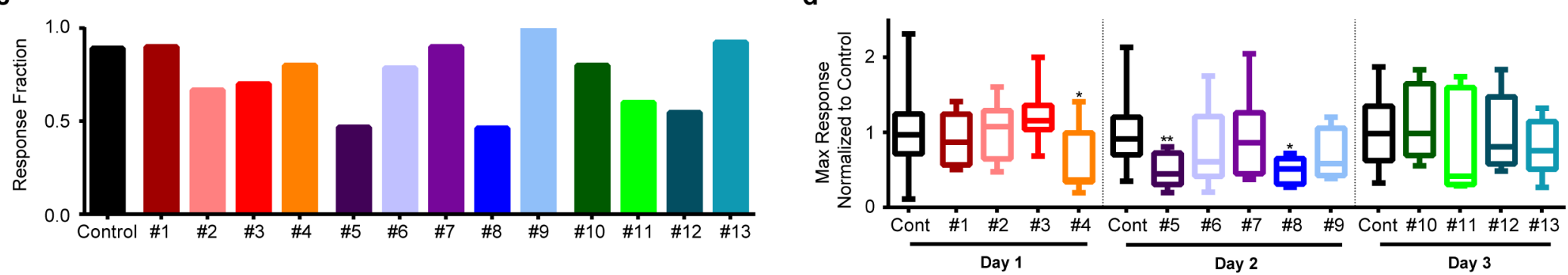

f
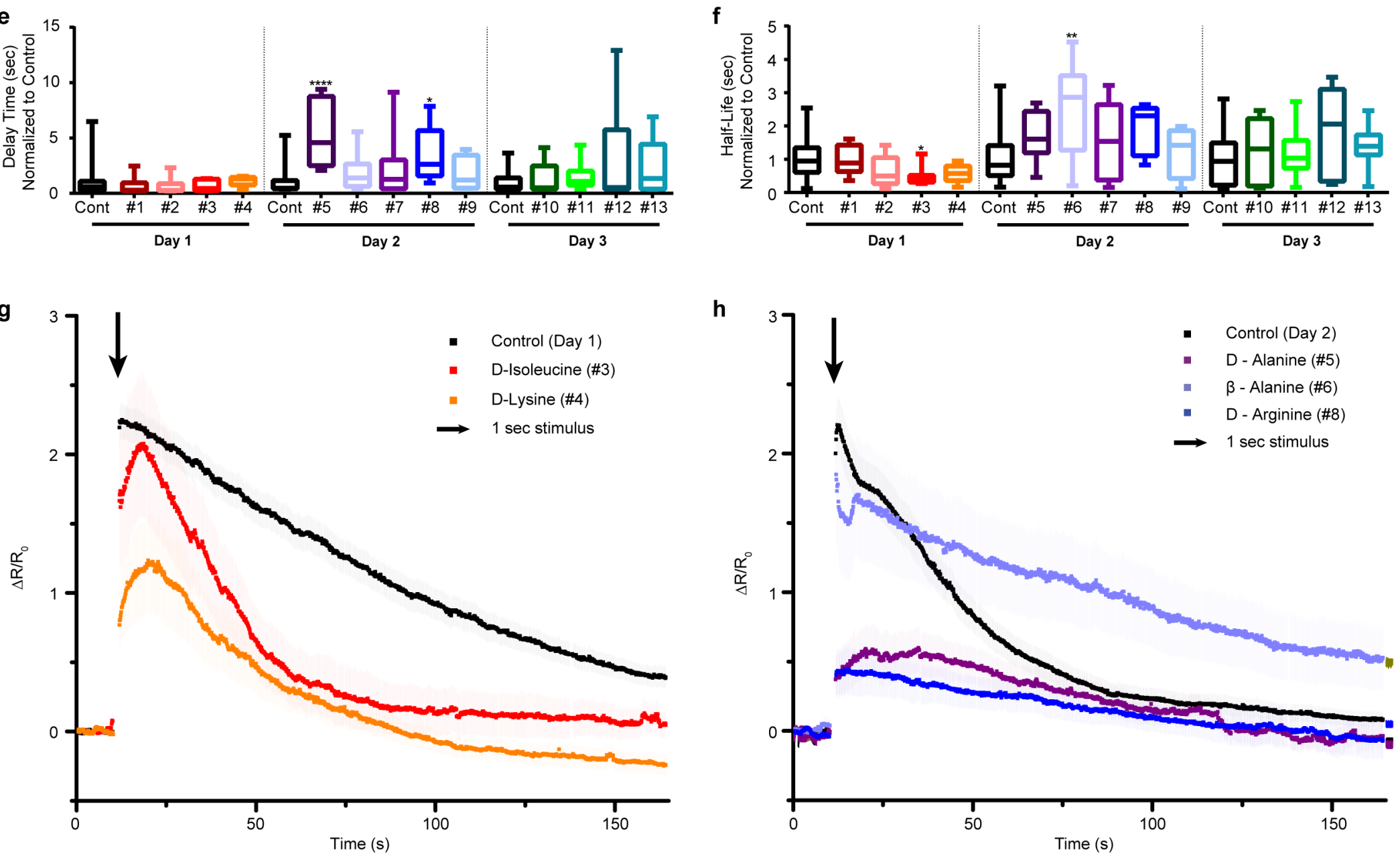

h

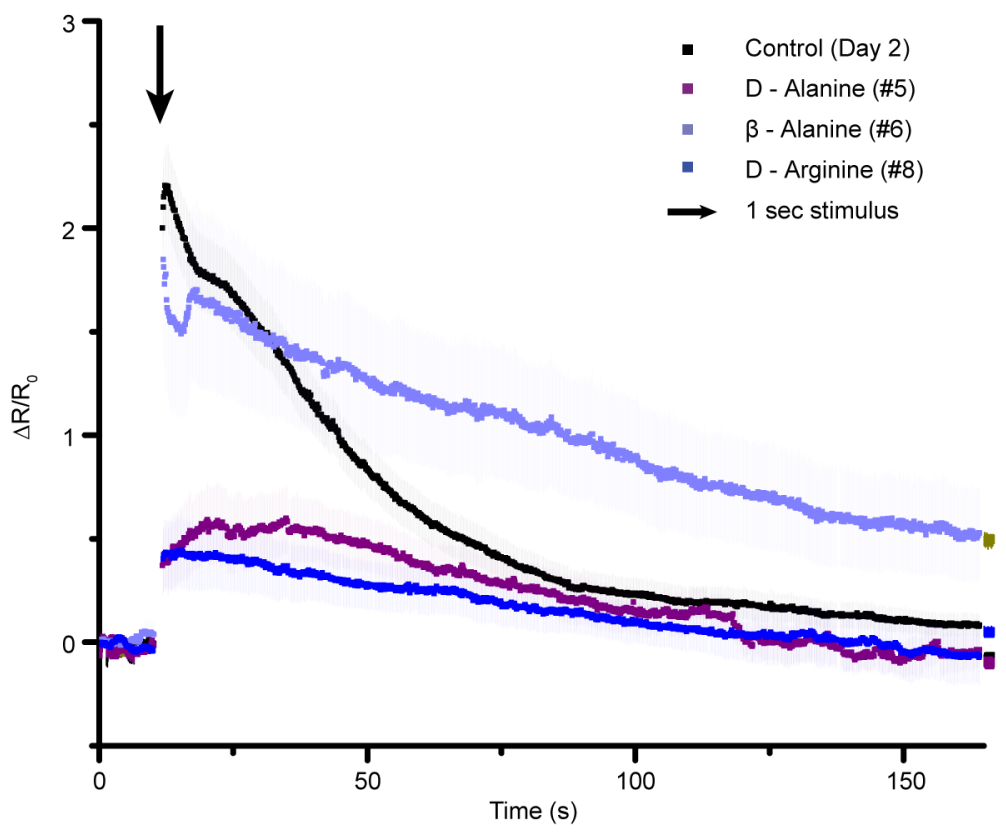

Cho et al., Figure 3. 
bioRxiv preprint doi: https://doi.org/10.1101/107318; this version posted February 10, 2017. The copyright holder for this preprint (which was not certified by peer review) is the author/funder, who has granted bioRxiv a license to display the preprint in perpetuity. It is made available under aCC-BY-NC-ND 4.0 International license.

a

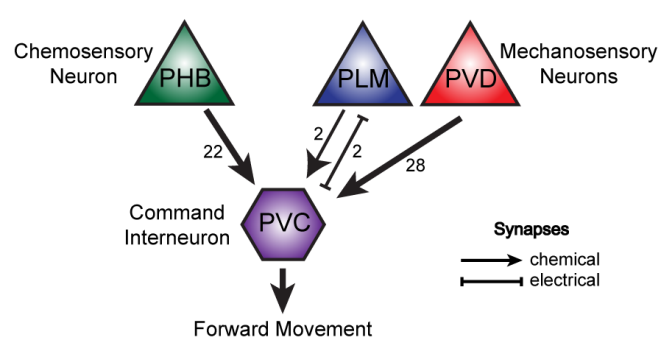

b

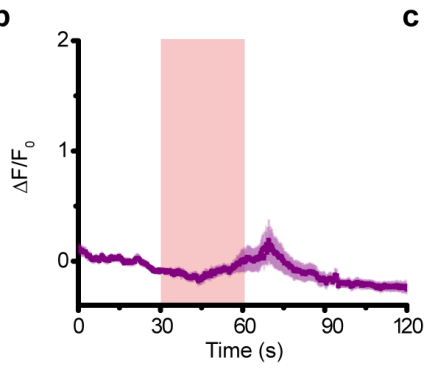

C
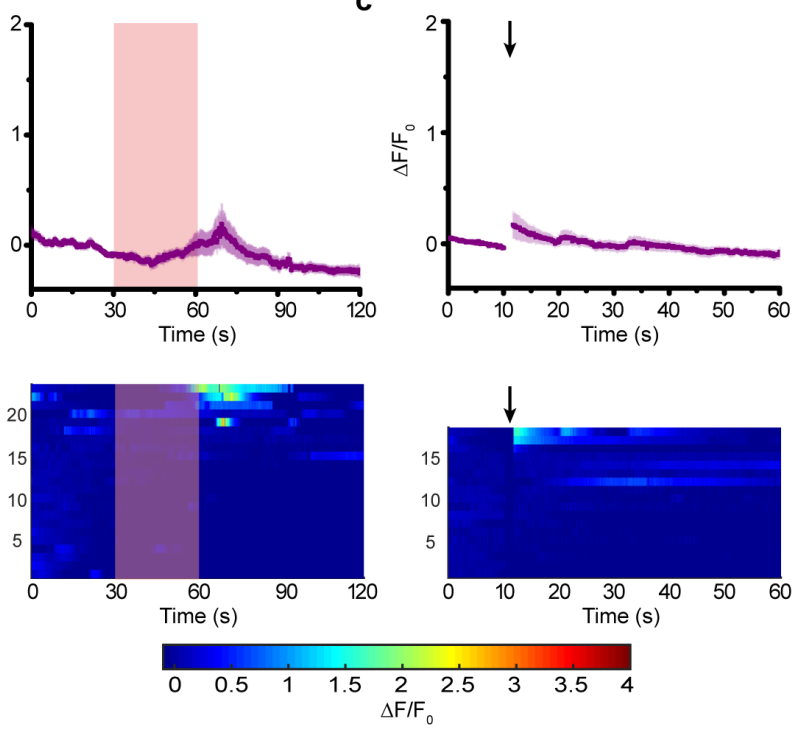

d

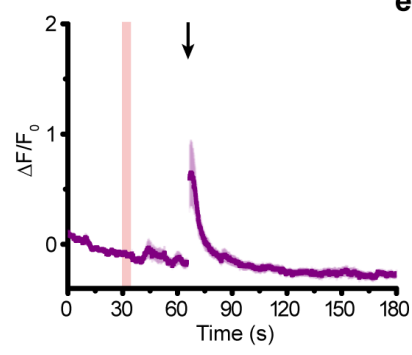

e
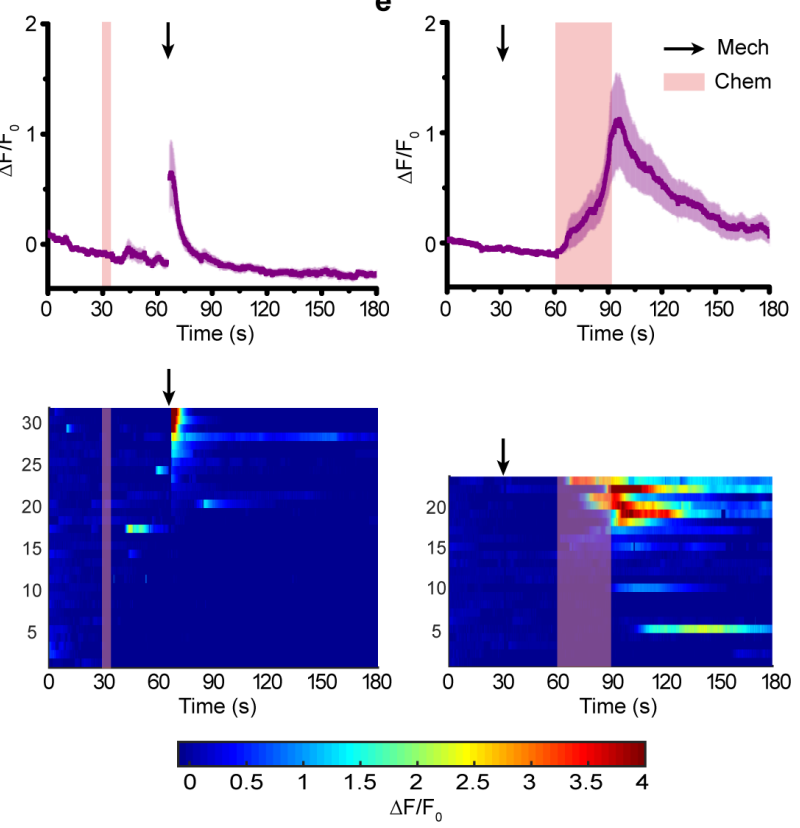

f

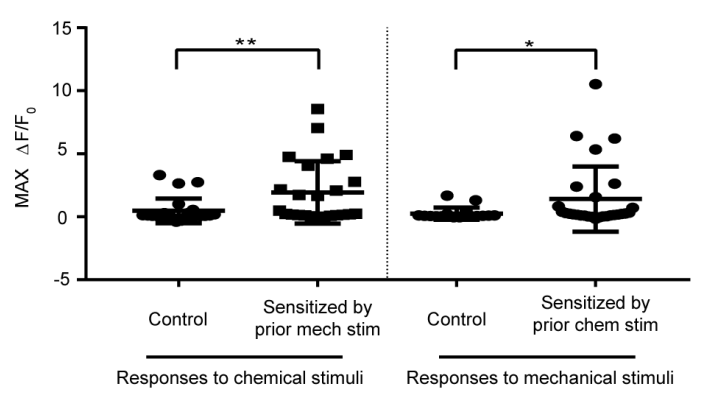

Cho et al., Figure 4. 


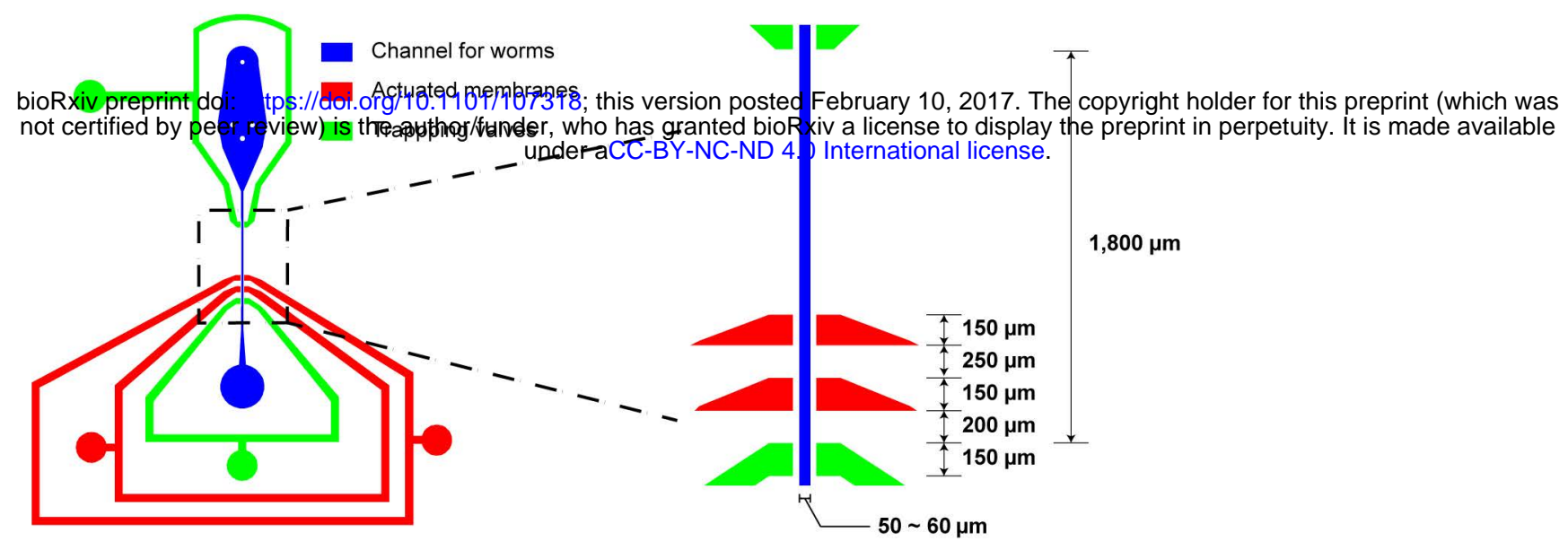

Cho et al., Supplementary Figure 1. 


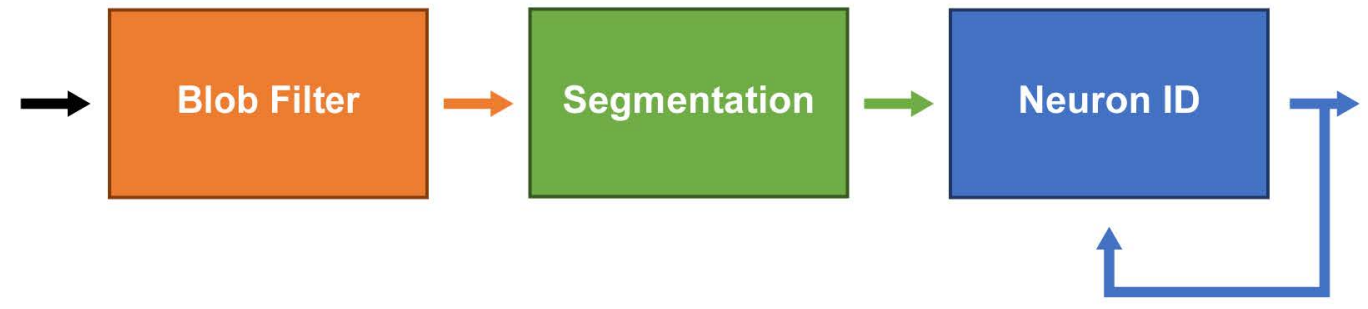

ROI Data Extraction

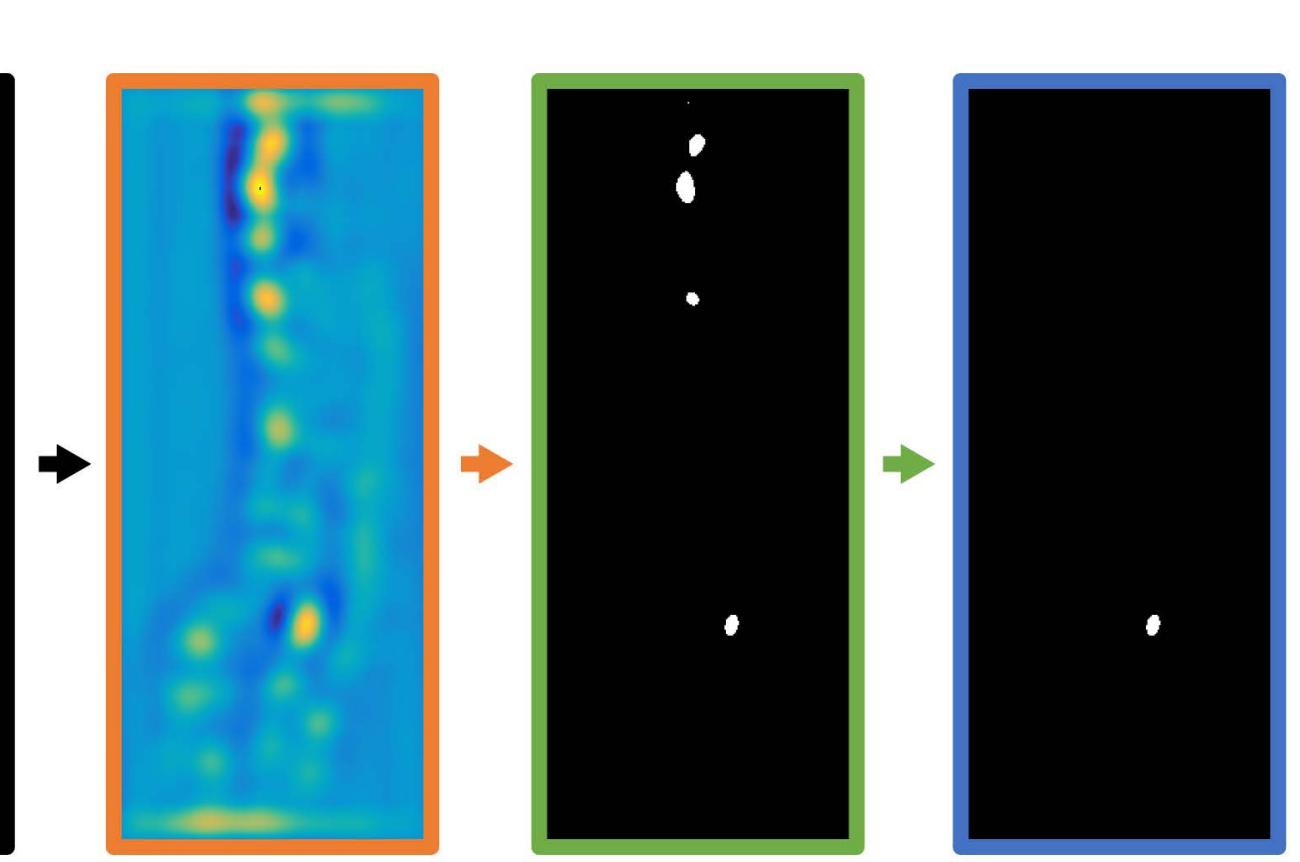

rgNeuron,

rgObjects

rgBlob

rgRaw $_{i}$ 
(1)

iv

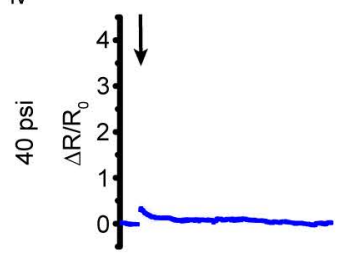

vii
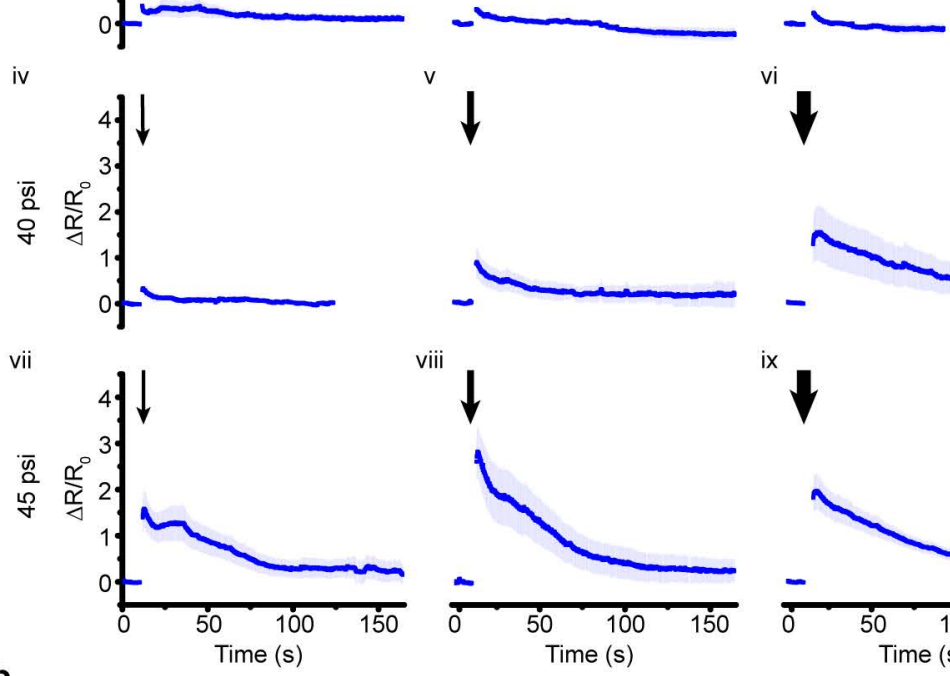

.

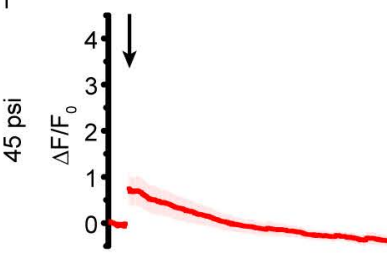

ii
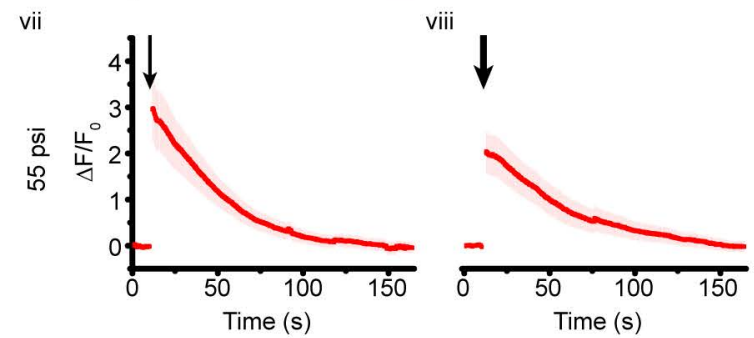
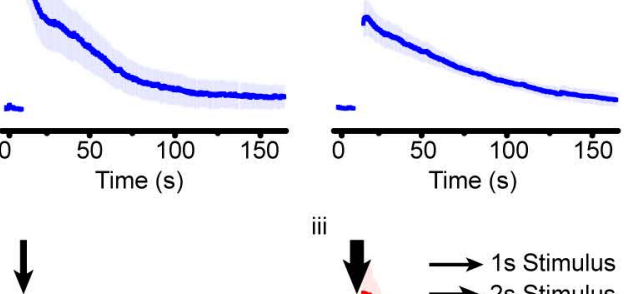

iii

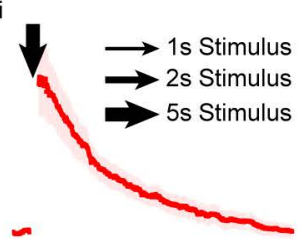

vi
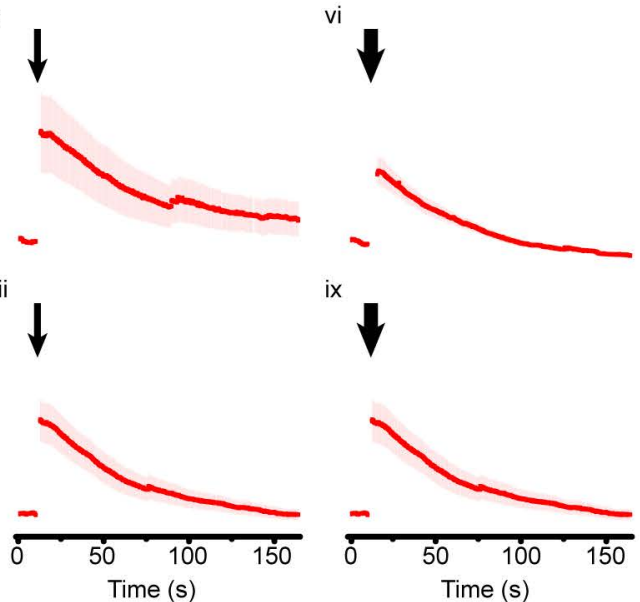

Cho et al., Supplementary Figure 4 . 

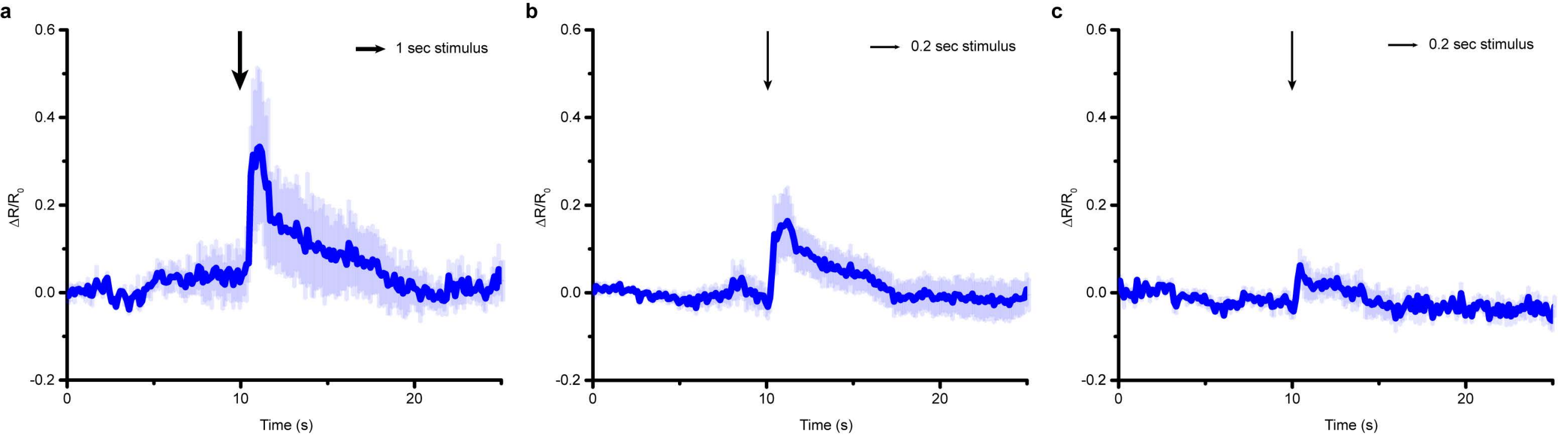

Cho et al., Supplementary Figure 5. 


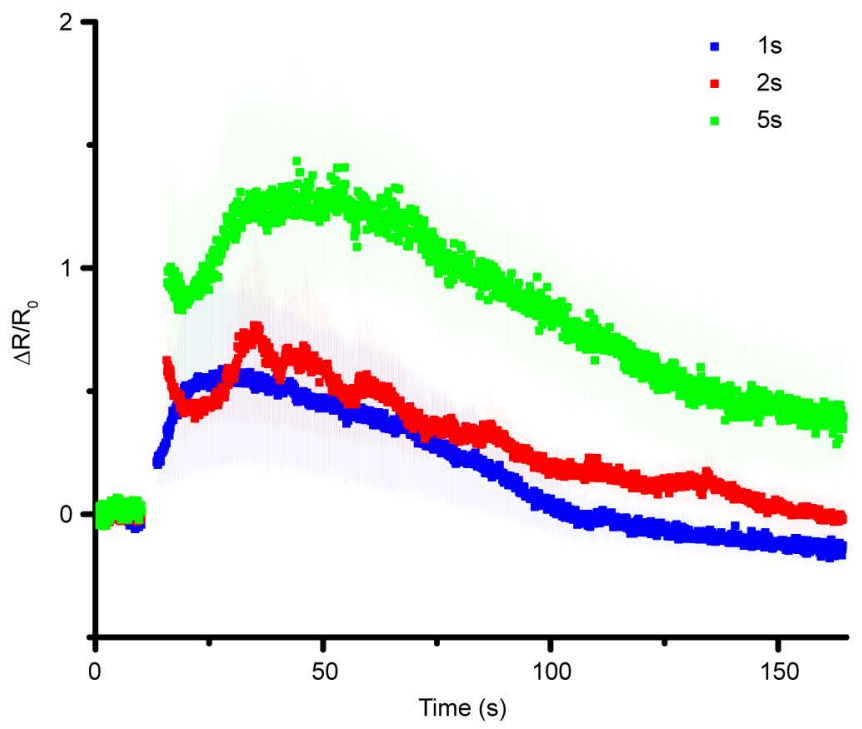

Cho et al., Supplementary Figure 6. 


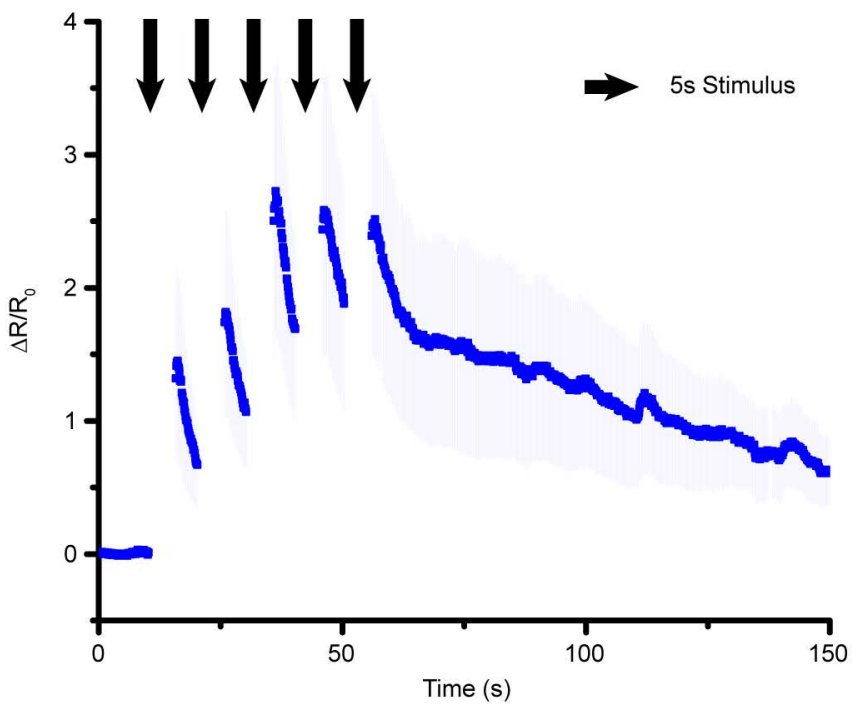

Cho et al., Supplementary Figure 7. 

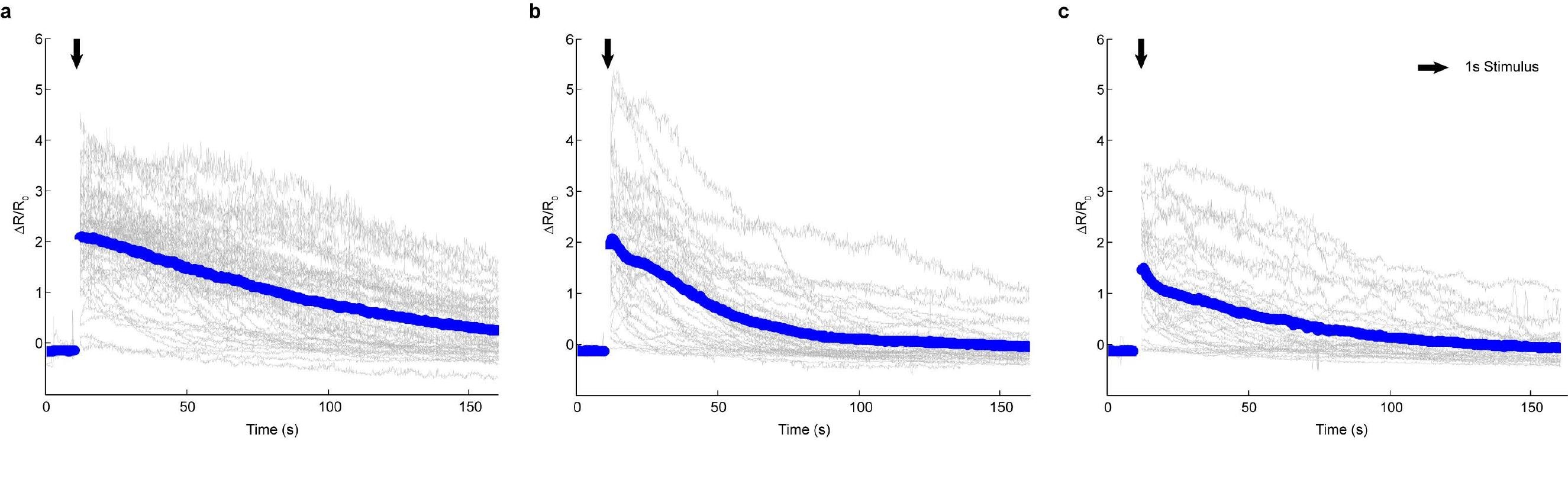

Cho et al., Supplementary Figure 8.

(n)
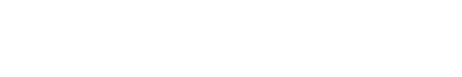


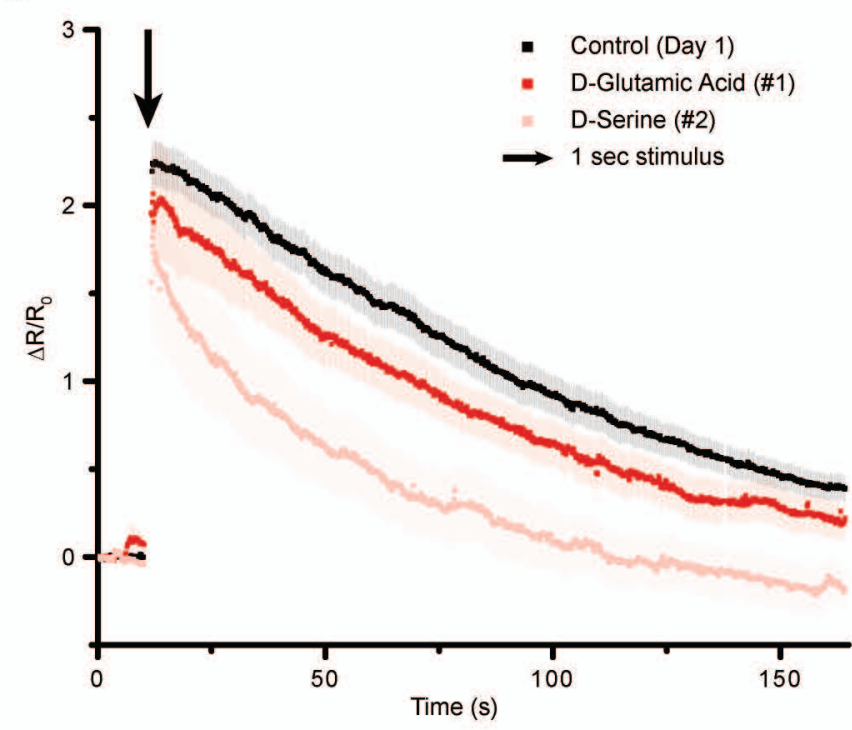

b

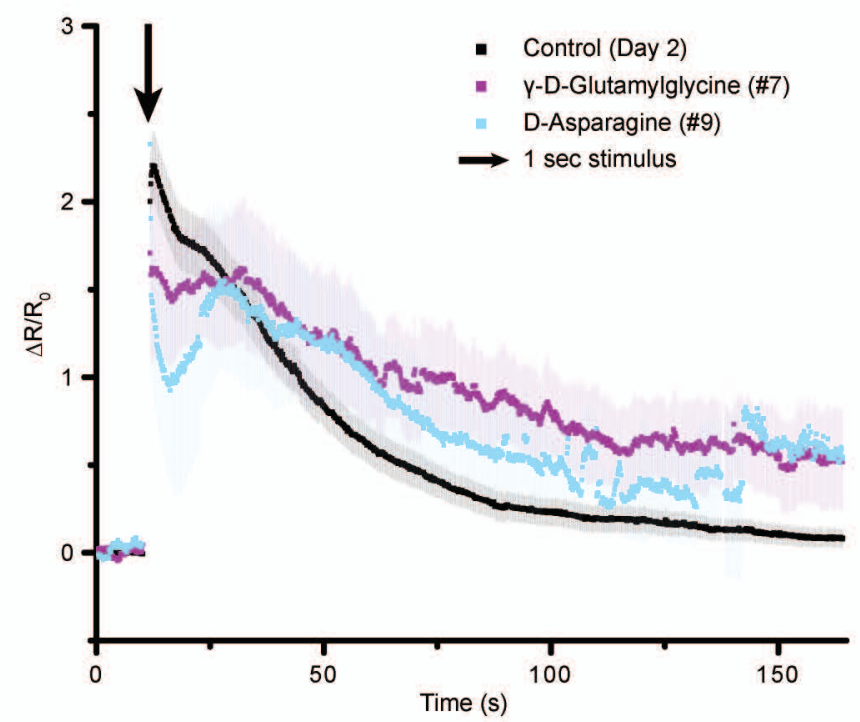

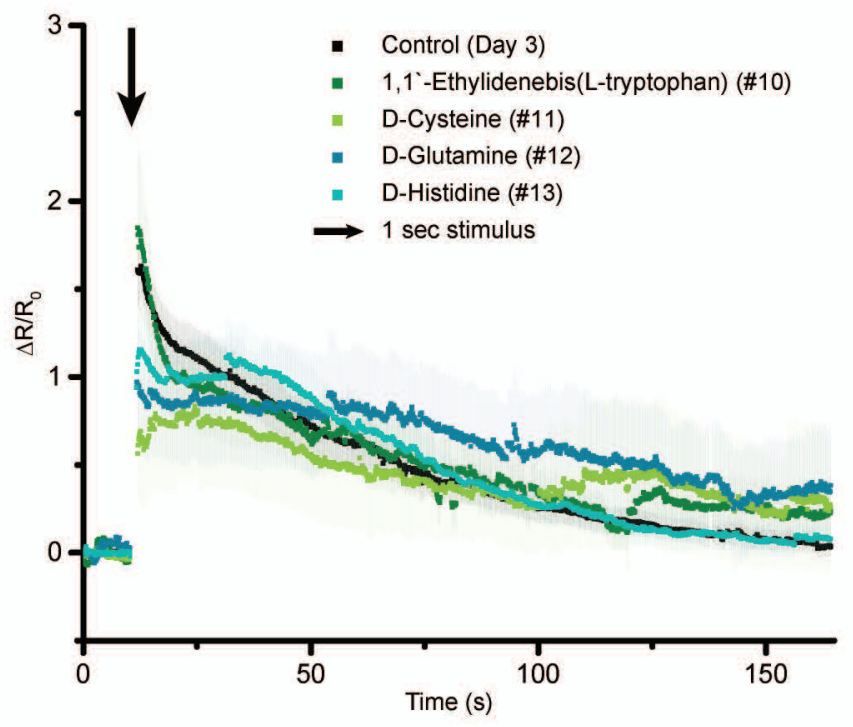

Cho et al., Supplementary Figure 9. 


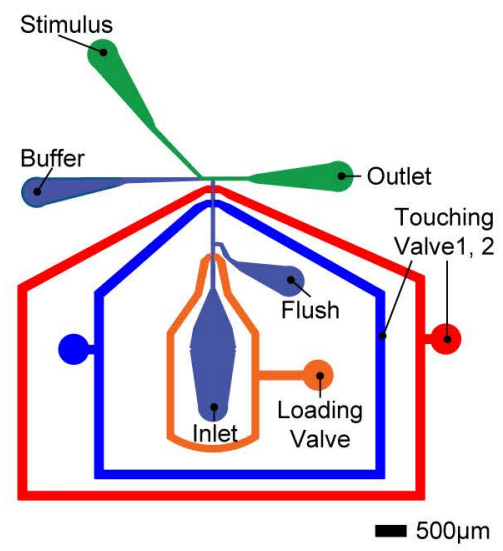

Cho et al., Supplementary Figure 10. 


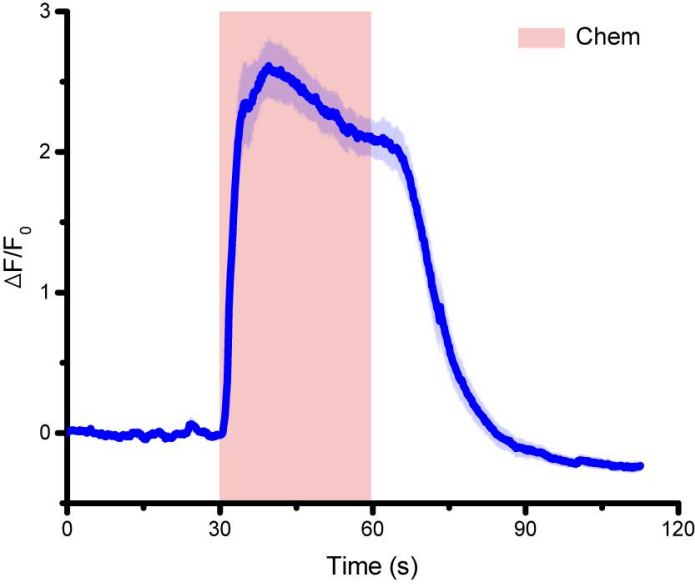




\begin{tabular}{|c|c|c|c|c|}
\hline Number & Name & Rationale & Sample size & $\begin{array}{l}\text { Number of } \\
\text { responding worms }\end{array}$ \\
\hline 1 & D-Glutamic acid & $\begin{array}{c}\text { Putative endogenous } \\
\text { ligand }\end{array}$ & 10 & 150 \\
\hline 2 & D-Serine & $\begin{array}{l}\text { Putative endogenous } \\
\text { ligand }\end{array}$ & 12 & 8 \\
\hline 3 & D-Isoleucine & D-Amino acid & 10 & 7 \\
\hline 4 & D-Lysine & D-Amino acid & 10 & 8 \\
\hline 5 & D-Alanine & D-Amino acid & 15 & 7 \\
\hline 6 & $\beta$-Alanine & Endogenous & 14 & 11 \\
\hline 7 & $\begin{array}{c}\gamma-\mathrm{D}- \\
\text { Glutamylglycine }\end{array}$ & D-Amino acid & 10 & 9 \\
\hline 8 & D-Arginine & D-Amino acid & 13 & 6 \\
\hline 9 & D-Asparagine & D-Amino acid & 4 & 4 \\
\hline 10 & $\begin{array}{c}\text { 1,1'-Ethylidene- } \\
\text { bis(L-tryptophan) }\end{array}$ & $\begin{array}{c}\text { Bioactive tryptophan } \\
\text { derivative }\end{array}$ & 10 & 8 \\
\hline 11 & D-Cysteine & D-Amino acid & 10 & 6 \\
\hline 12 & D-Glutamine & D-Amino acid & 11 & 6 \\
\hline 13 & D-Histidine & D-Amino acid & 13 & 12 \\
\hline
\end{tabular}

Cho et al., Supplementary Table 1. 